\title{
Multi-Cell MIMO Cooperative Networks: A New Look at Interference
}

\author{
David Gesbert, Stephen Hanly, Howard Huang, Shlomo Shamai Shitz, Osvaldo Simeone, and Wei Yu
}

\begin{abstract}
This paper presents an overview of the theory and currently known techniques for multi-cell MIMO (multiple input multiple output) cooperation in wireless networks. In dense networks where interference emerges as the key capacitylimiting factor, multi-cell cooperation can dramatically improve the system performance. Remarkably, such techniques literally exploit inter-cell interference by allowing the user data to be jointly processed by several interfering base stations, thus mimicking the benefits of a large virtual MIMO array. Multicell MIMO cooperation concepts are examined from different perspectives, including an examination of the fundamental information-theoretic limits, a review of the coding and signal processing algorithmic developments, and, going beyond that, consideration of very practical issues related to scalability and system-level integration. A few promising and quite fundamental research avenues are also suggested.
\end{abstract}

Index Terms-Cooperation, MIMO, cellular networks, relays, interference, beamforming, coordination, multi-cell, distributed.

\section{INTRODUCTION}

A. Dealing with interference: conventional and novel approaches

$\mathbf{F}$ ADING and interference are the two key challenges faced by designers of mobile communication systems. While fading puts limits on the coverage and reliability of any point-to-point wireless connection, e.g., between a base station and a mobile terminal, interference restricts the reusability of the spectral resource (time, frequency slots, codes, etc.) in space, thus limiting the overall spectral efficiency expressed in $\mathrm{bits} / \mathrm{sec} / \mathrm{Hz} / \mathrm{base}$ station. At least, so has been the conventional view until recent findings in the area of cooperative transmission. Two basic scenarios are envisioned for cooperation in wireless networks. The first one assumes a (virtual) MIMO model for cooperative transmission over otherwise interfering links and will be the focus of this paper, while in the second relays are exploited. There exist interesting conceptual bridges

Manuscript received 10 January 2010; revised 1 July 2010. The review of this tutorial article was coordinated by Senior Editor Pamela Cosman.

D. Gesbert is with EURECOM, 06904 Sophia Antipolis, France (e-mail: gesbert@eurecom.fr).

S. Hanly is with the National University of Singapore Department of Electrical and Computer Engineering (e-mail: elehsv@nus.edu.sg).

H. Huang is with Bell Labs, Alcatel-Lucent, NJ., USA (e-mail: hchuang@alcatel-lucent.com).

S. Shamai Shitz is with Technion, Israel (e-mail: sshlomo@ee.technion.ac.il).

O. Simeone is with the Center for Wireless Communication and Signal Processing Research (CWCSPR) New Jersey Institute of Technology (NJIT) (e-mail: osvaldo.simeone@njit.edu).

Wei $\mathrm{Yu}$ is with The Edward S. Rogers Sr. Department of Electrical and Computer Engineering, University of Toronto (e-mail: weiyu@comm.utoronto.ca).

Digital Object Identifier 10.1109/JSAC.2010.101202. between the two setups however, as will be made clearer in Section III and beyond.

Relay-based cooperative techniques try to mitigate detrimental propagation conditions from a transmitter to a receiver by allowing communication to take place via a third party device (mobile or base) acting as a relay. Initially developed relay-based cooperative transmission protocols have proved to be instrumental in mitigating fading effects (both path loss and multipath related) in point-to-point and point-to-multipoint communications. So-called amplify-forward, decode-forward, compress-forward cooperation schemes exploit available relay nodes to offer a powerful extra diversity dimension [1].

While conventional diversity and relaying schemes greatly improve the link-level performance and reliability, they do little to increase the quality of service to users placed in severe inter-cell interference-dominated areas, such as the cell boundary areas of current cellular networks. Instead, interference should be dealt with using specific tools such as "virtual" or "network" MIMO, so as to maximize the number of co-channel links that can coexist with acceptable quality of service. In the high SNR regime (achieved in, say, a small cell scenario), this figure of merit corresponds to the maximum number of concurrent interference-free transmissions and is referred to as the multiplexing gain of the network, or the number of degrees of freedom in the information-theoretic terminology.

The conventional non-cooperative approach to interference, via spatial reuse partitioning, prevents the reuse of any spectral resource within a certain cluster of cells. Typically, the frequency re-use factor is much less than unity, so that the level of co-channel interference is low. Thus, interference is controlled by fixing the frequency reuse pattern and the maximum power spectral density levels of each base station. Current designs do allow for full frequency re-use in each cell (typically for Code Division Multiple Access (CDMA) or frequency hopping spread spectrum systems) but this results in very severe interference conditions at the cell edge, causing a significant data rate drop at the terminals and a strong lack of fairness across cell users. Some interference mitigation is offered by limited inter-cell coordination, which is conventionally restricted to scheduling or user assignment mechanisms (e.g. cell breathing) or soft handover techniques. Inter-cell interference is treated as noise at the receiver side and is handled by resorting to improved point-to-point communications between the base station (BS) and the mobile station (MS), using efficient coding and/or single-link multiple-antenna techniques [2]. This approach to dealing with interference may be characterized as passive. 
In contrast, the emerging view on network design advocates a more proactive treatment of interference, which can be accomplished through some form of interference-aware multi-cell coordination, at the base station side. Although the complexity associated with the coordination protocols can vary greatly, the underlying principle is the same: Base stations no longer tune separately their physical and link/MAC layer parameters (power level, time slot, subcarrier usage, beamforming coefficients etc.) or decode independently of one another, but instead coordinate their coding or decoding operations on the basis of global channel state and user data information exchanged over backhaul links among several cells. Coordination protocols can exploit pre-existing finite capacity backhaul links (e.g., 802.16 WiMax, 4G LTE) or may require a design upgrade to accommodate the extra information sharing overhead. There are several possible degrees of cooperation, offering a trade-off between performance gains and the amount of overhead placed on the backhaul and over-the-air feedback channels. The different possible levels of cooperation are illustrated in detail in Section II.

\section{B. From multi-user to multi-cell MIMO}

The history of base station cooperation can be traced back to previous decades, with the concept of macroscopic diversity whereby one or more mobiles communicate their messages through multiple surrounding base stations to provide diversity against long-term and short-term fading. In conventional CDMA networks, soft-handoff allows a mobile to communicate simultaneously with several base stations, and selection diversity is used to select the best of these connections at any given time. Such selection diversity increases both coverage and capacity [3], and combined with power control, it allows full frequency re-use in each cell. However, full frequency re-use comes at a price: CDMA capacity is then critically constrained by inter-cell interference, and the per-cell capacity in a network of interfering cells is much less than that of a single isolated cell. This reduction in capacity is measured by the so-called "f-factor" [4]. We will see that full base station cooperation essentially removes this interference penalty.

By "full base station cooperation" we mean that all base stations are effectively connected to a central processing site, as depicted in Figure 1 for the downlink scenario. On the downlink, the network is effectively a MIMO broadcast channel with distributed antennas. First steps toward full base station cooperation were taken in [5], [6], [7] for the uplink, which is effectively a MIMO multiple access channel. In these works, the base stations cooperate to decode each user. In [6], the model is a CDMA network, with single-user matched filter (SUMF) decoding, but the received signals from a mobile, at each base station, are maximal ratio combined before decoding. With such a global receiver there are no wasted signals causing pure interference: All received signals carry useful information for the global decoder, and hence interference is exploited. In [6], it is shown that with the optimal power control, such base station cooperation eliminates the inter-cell interference penalty. In other words, a network of interfering cells has the same per-cell capacity (in numbers of users) as a single, isolated cell. This result

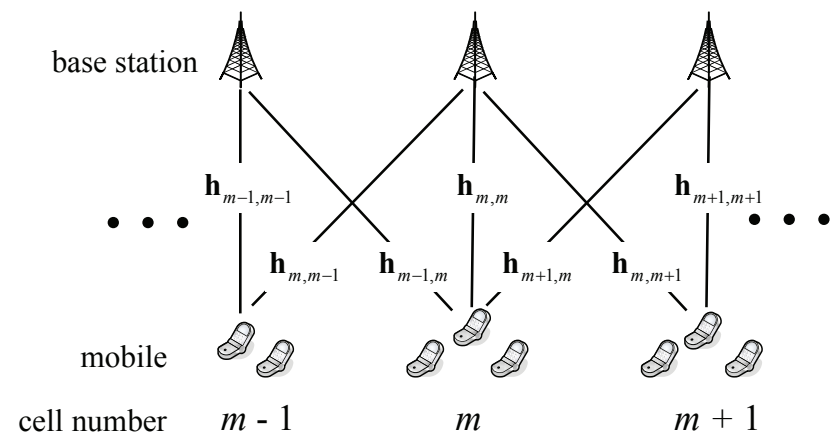

Fig. 1. A linear Wyner-type model with inter-cell interference spans $L_{\ell}=$ $L_{r}=1$ and $K=3$ MSs per cell.

was extended to CDMA networks with more sophisticated multi-user receivers (decorrelator and MMSE receivers) in [7]. Again, the interference is fully eliminated and the achievable number of simultaneous users is same as if the cells were isolated from each other, although in this case the per-cell capacity benefits further from the more sophisticated multiuser detection.

The story is not so clear-cut if we consider more fundamental, information-theoretic models, where particular physical layers (e.g., CDMA) or receiver structures, are not assumed a priori. Such information-theoretic results will be surveyed in Section III. However, similar conclusions do hold at high SNR, in terms of the degrees of freedom, as will be seen. Pioneering work on the information-theoretic capacity of the uplink of cellular networks with full base station cooperation was done in the early 90s [8], [9]. In these works, it was shown that with full base station cooperation, the traditional approach of frequency re-use partitioning is inherently suboptimal. Wyner [9] introduced a linear array model, and a hexagonal cell model, which have become known as Wyner models of cellular systems, and these are very tractable for informationtheoretic analysis. In [8], it was shown that at high SNR, the capacity of a cellular system with fractional frequency re-use is less than a system with full frequency re-use, by exactly the re-use factor. This is equivalent to saying that full base station cooperation reduces the inter-cell interference penalty (or "f-factor") to zero.

Although rich in content and ideas, [8], [9] stopped just short of spelling out the connections between the multi-user multi-cell channel and the MIMO channel. Communication over the spatial modes of the point-to-point MIMO channel (so-called spatial multiplexing) was formalized later in the mid 90s in [10], [11], then gradually extended to multi-user (MU-) MIMO channels. It was at that stage only that the downlink of the multi-cell cooperative channel, first investigated for the downlink in 2001 [12], was recognized to be almost identical to the so-called broadcast MIMO channel, if one ignores the power constraint at the individual base stations. On the uplink, there is no difference between ideal ${ }^{1}$ multi-cell MIMO decoding and decoding over a multi-user MIMO channel.

\footnotetext{
${ }^{1}$ An ideal multi-cell MIMO channel is one where all base stations are connected via infinite capacity backhaul links.
} 
Thus, a network of $M$ ideally connected $J$-antenna base stations can serve a total of $M J$ terminals in an interferencefree manner simultaneously, regardless of how strong the interference is. To achieve this remarkable result, multi-user spatial precoding and decoding is involved on the downlink and uplink respectively, much akin to techniques used over the MU-MIMO channel [13]. To date, progress in the area of multi-cell MIMO cooperation continues to parallel that realized in the more general MU-MIMO area. Nevertheless, this domain of communications provides specific and tough scientific challenges to communication theorists, although, remarkably, it is already being considered within industry and standardization fora.

\section{Challenges of multi-cell MIMO}

Despite their promise, multi-cell MIMO systems still pose a number of challenges both theoretical and practical, several of which are described in this paper. First, a thorough understanding of the information-theoretic capacity of multi-cell MIMO system accounting for fading and path loss effects, even with an ideal backhaul, is yet to be obtained. As reviewed in this paper, capacity results exist for simplified interference models. Such results provide intuition for the general performance behavior but are difficult to extend to general channel models. Second, as multi-cell channels may involve a large number of antennas and users, algorithm development work is required to reduce the complexity of currently proposed precoding and decoding schemes. Optimal precoding over the broadcast (downlink) MIMO channel as well as optimal joint decoding over the uplink involve non-linear computationally intensive operations [14], [15] which scale poorly with the size of the network. Third, the equivalence between multi-cell systems and MIMO systems only holds in the case of ideal backhaul conditions. Practical cooperation schemes must operate within the constraints of finite capacity, finite latency communications links between base stations.

Deriving good theoretic performance bounds for MIMO cooperation over a channel with limited information exchange capability between the cooperating transceivers is a difficult task. As shown in this paper some results are available for a few simplified network models. From a practical point-ofview, a major research goal is to find good signal processing and coding techniques that approach ideal cooperative gains while relying on mostly local channel state information and local user data. This problem, referred to here as distributed cooperation, is as challenging as it is important. Efficient partial feedback representation methods building on classical MIMO research [16] are also desirable. From a systemlevel perspective, simulations indicate that substantial gains in capacity and increased fairness across cell locations will be accrued from the adoption of multi-cell MIMO techniques. Yet, a number of important practical issues must be addressed before a very realistic assessment of system gains can be made, such as the impact of imperfect synchronization between base stations, imperfect channel estimation at the receiver side, and network latency. Such aspects are addressed at the end of the paper along with a review of current field experiments.

\section{Scope and organization of paper}

The theoretical treatments of interference-limited channels on the one hand, and of cooperation protocols on the other hand, are still maturing, mostly due to the inherent complexity of the problem. Nevertheless, the literature in these areas has grown to be extremely rich. For this reason, we do not attempt complete coverage of those domains of research. Instead, we focus on the adaptation of multi-antenna processing principles to the context of multi-cell cooperation. We refer to the obtained framework as multi-cell processing (MCP).

In Section II, we begin with the mathematical models for the network and signals, and the way that information is exchanged between the cells. Basic notations for multi-cell cooperation are given. Next, in Section III, key informationtheoretic results are surveyed that establish closed-form expressions for sum rate bounds for several important interference and backhaul models. In Sec. IV, the focus is the design of practical MCP techniques, assuming an ideal backhaul. Sec. $\mathrm{V}$ deals with the problem of finite capacity backhaul and considers the feasibility of scalable and distributed MIMO cooperation, using such concepts as partial feedback, distributed optimization, Turbo base stations, and clustering. Sec. VI addresses system-level implementation issues due to expected imperfections at the physical layer. Initial tests with prototypes are reported. Finally, Sec. VII provides perspectives and suggestions for promising research avenues in this area.

Throughout this paper we adopt the following notations: $[\mathbf{x}]_{k}$ represents the $k$ th element of vector $k ; \mathbf{1}_{N}$, and $\mathbf{0}_{N}$ are $N \times 1$ vectors of all ones and all zeros, respectively; $[a, b]$, where $a \leq b$ are integer, is the interval $[a, \ldots, b] ;(\cdot)^{\dagger}$ represent the conjugate transpose of its argument. I denotes the identity matrix.

\section{Modelling MULti-CELl COOPERATiON}

\section{A. System model}

We consider a multi-cell network comprising $M$ cooperating base stations assigned with the same carrier frequency. Each cell serves $K$ users. The base stations are equipped with $J$ antennas each. Due to lack of space, we mostly focus on base station-side interference control: The users have single-antenna terminals, unless otherwise specified. Multiple antenna terminals can be considered to allow for the spatial multiplexing of mutiple data streams per user, or to give userside multi-cell interference cancellation capability. The latter turns out to be useful especially in the context of interference coordination. This scenario is addressed in Section IV, but is otherwise excluded. The base stations can assume any geometry, however, strongly structured cell models can help the theoretical analysis of cooperation, as is discussed in Section III.

In the uplink, the received signal at the $m$ th BS, $m \in[1, M]$ can be written as

$$
\mathbf{y}_{m}=\sum_{l=1}^{M} \sum_{k=1}^{K} \mathbf{h}_{m, l, k} x_{l, k}+\mathbf{z}_{m},
$$

where $x_{l, k}$ is the symbol transmitted by the $k$-th MS in the $l$ th cell, $\mathbf{h}_{m, l, k}$ denotes the $J$ element channel vector from the $k$-th user of cell $l$ towards the $m$ th $\mathrm{BS}, \mathbf{z}$ is the noise vector 
containing additive noise and any inter-cell interference not accounted for by the $M$ cooperating cells alone, for instance if the networks features more than $M$ BSs.

The model for the downlink can be easily obtained from the one above. We will reuse some symbols, but their meaning will be clear from the context. The signal received at the $k$-th user in the $m$-th cell is written as:

$$
y_{m, k}=\sum_{l=1}^{M} \mathbf{h}_{l, m, k}^{\dagger} \mathbf{x}_{l}+z_{m},
$$

where $\mathbf{x}_{l}$ is the transmitter signal vector with $J$ elements transmitted from the $l$-th BS containing possibly precoded (beamformed) information symbols for several users. Note that, as a convention, the downlink channel vector from the $l$-th BS towards the $k$-th user in the $m$-th cell is denoted by the complex conjugated form of the corresponding uplink channel $\mathbf{h}_{l, m, k}$. This is done to allow the exploration of interesting duality results between uplink and downlink, as seen in Sections III and IV. Note that this represents a convenient writing convention rather than an actual assumption on physical reciprocity of the uplink and downlink channels. Where/if such an assumption of reciprocity (e.g., TDD system) is actually needed will be made clear in the paper.

\section{B. The different levels of multi-cell cooperation}

In this paper we distinguish simpler forms of multi-cell coordination from those requiring a greater level of information sharing between cells.

1) Interference coordination: The performance of current cellular networks can already be improved if the BSs share the channel state information of both the direct and interfering links, obtained from the users via feedback channels (see Fig 2). The availability of channel state information (CSI) allows BSs to coordinate in their signaling strategies, such as power allocation and beamforming directions, in addition to user scheduling in time and frequency. This basic level of coordination requires a relatively modest amount of backhaul communication and can be quite powerful if enough users co-exist in the system (multi-user diversity) [17]. No sharing of transmission data, or signal-level synchronization between the base stations is necessary. We refer to such schemes as interference-coordination. In this case, the downlink signal at the $l$-th base, $\mathbf{x}_{l}$, is a combination of symbols intended for its $K$ users alone.

2) MIMO cooperation: On the other hand, when base stations are linked by high-capacity delay-free links, they can share not only channel state information, but also the full data signals of their respective users (see Fig. 3). A more powerful form of cooperation can be achieved. In this scenario, the concept of an individual serving base for one terminal disappears since the network as a whole, or at least a group of cells, is serving the user. The combined use of several BS antennas belonging to different cells to send or receive multiple user data streams mimicks transmission over a MIMO channel and is referred to here as MIMO cooperation. In principle, MIMO cooperation transforms the multi-cell network into a multiuser MIMO (MU-MIMO) channel for which all propagation links (including interfering ones) are exploited to carry useful

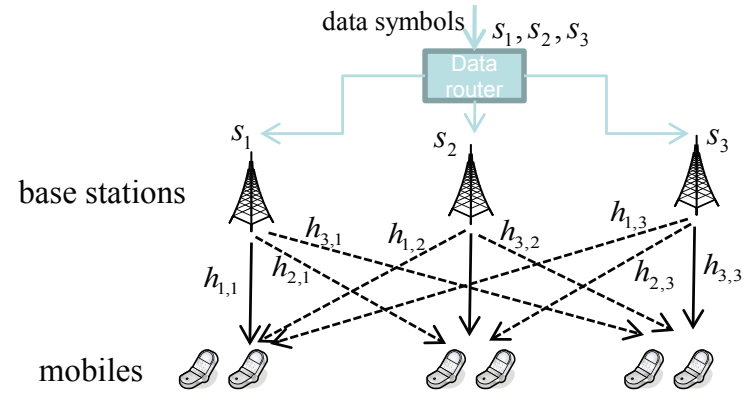

Fig. 2. Illustration of interference coordination for the downlink. The BSs acquire and exchange channel state information (but not the data symbols) pertaining to all relevant direct and interfering links, so as to optimize jointly their transmission parameters (time-frequency scheduling, power level, beamforming

data, upon appropriate precoding/decoding. In this case, the downlink signal, $\mathbf{x}_{l}$, is a combination of symbols intended for all $M K$ users. In contrast, interference-coordination schemes try to mitigate the generated interference, but they cannot really exploit it. For instance, beamforming may be used in each cell if the base stations are equipped with multiple antennas. In this case the beams typically try to strike a compromise between eliminating the inter-cell interference and maximizing the received energy to/from the user within the cell of interest. Ideally the choice of such beams across multiple cells is coordinated.

Although some interference-coordination ideas are promising, they are touched upon rather briefly in this paper, mainly in Section IV. MIMO cooperation schemes are the main focus of our attention.

3) Rate-limited MIMO cooperation: In the intermediate case, the base stations are linked by limited-capacity backhaul links. Typically, channel state information is shared first, then only a substream of user data or a quantized version of the antenna signals are shared among the base stations, which allows partial interference cancellation. Such hybrid scenarios are investigated from an information-theoretic point of view in Section III, then from an algorithmic perspective in Sections IV and $\mathrm{V}$.

4) Relay-assisted cooperation: Instead of cooperating through backhaul links, it is also possible to consider channel models in which a separate relay node is available to assist the direct communication within each cell. Relay communication is relevant to the multi-cell MIMO network because it can be beneficial not only in strengthening the effective direct channel gain between the BS and the remote users, but also in helping with intercell interference mitigation. Relay enabled cooperation is studied in Sec. III-E.

\section{CAPACITY RESUlTS FOR MULTI-CELL MIMO COOPERATION}

In this section, we address the impact of cooperation on cellular systems from an information-theoretic standpoint. We mostly focus on the performance of MCP but we also consider 


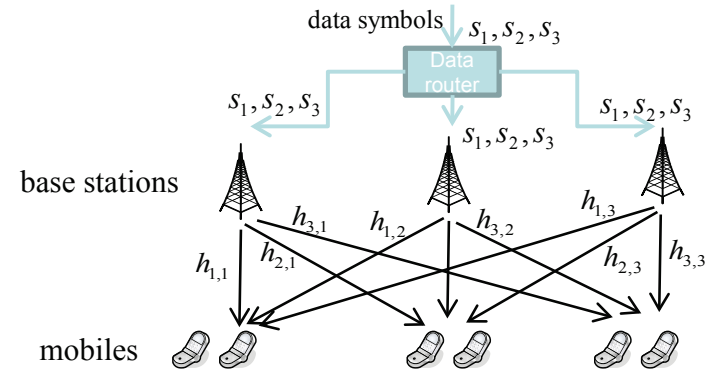

Fig. 3. Illustration of multi-cell MIMO for the downlink. The BSs, each equipped with $J$ antennas, acquire and share channel state information and user data, so as to mimic the behavior of a large MIMO array with $M J$ antennas

the interplay of such techniques with cooperation in the form of relaying at the Mobile Stations (MSs) level.

\section{A. Introduction}

The analysis of MCP was started in the early works [8][9] for the uplink and [12] for the downlink. The analysis in these works is based on the assumption that the BSs are connected via unrestricted backhaul links (error-free and unlimited capacity) to a Central Processor (CP) and focuses on models that, in information theoretic terms, can be seen as symmetric Gaussian multiple-access or broadcast interference channels. In these models, typically referred to as Wyner-type models, a number of users per cell is served by a singleantenna BS, as in a multiple access or broadcast channel, and interference takes place only between adjacent cells, as in partially connected interference networks [18]. Both models where cells are arranged on a line or in a more conventional bidimensional geometry can be considered, where the first class may model systems deployed along a highway or long corridor (see [19] for an implementation-based study), while the second applies to more general scenarios.

In this section, we consider the multi-cell MIMO scenario of Fig. 3 (i.e. with backhaul links allowing for some exchange of CSI and data symbols information), however we will focus on simplified cellular models that extend the Wyner-type models considered in the initial works [8], [9], [12]. Specifically, we consider the presence of limited-capacity and limitedconnectivity backhaul links, fading channels and the interplay of MCP with relaying. We will focus on the per-cell sumrate as the criterion of interest. It should be noted that, while the considered models capture some of the main features and practical constraints of real cellular systems, such as the locality of interference and constrained backhaul links, other features such as user-dependent path loss are not accounted for (see, e.g., [20]). Therefore, the models at hand can be seen as useful simplifications of real cellular settings, that enable insights and intuition to be obtained via analysis. It should be also noted that the use of sum rate as a system metric may mask other interesting features of multi-cell cooperation such as improving the balancing of user's quality of service from cell center to cell edge.

\section{B. The Linear Wyner Model}

In this section, we review a basic system model for multiple cell networks introduced in [8], [9]. We focus the attention on linear Wyner-type models, as done in the original works. Extension of the given results to planar models is possible, though not always straightforward and we refer to [21] for further discussion on this point. An extension of the model to include relays is discussed in Section III-E of the present paper. A linear Wyner-type model is sketched in Fig. 1. We now present the corresponding signal models for uplink and downlink.

1) Uplink: A general linear Wyner-type model is characterized by $M$ cells arranged on a line (as for a highway or corridor), each equipped with a single-antenna $(J=1)$ base station (BS) and $K$ single-antenna MSs. In this class of models, inter-cell interference at a given BS is limited to $L_{\ell}$ BSs on the left and $L_{r}$ on the right. Considering the uplink, the received signal (1) at the $m$ th $\mathrm{BS}, m \in[1, M]$, at a given time instant $t \in[1, n]$ ( $n$ is the size of the transmitted block) can then be specialized as

$$
y_{m}(t)=\sum_{l=-L_{r}}^{L_{\ell}} \mathbf{h}_{m, m-l}^{T}(t) \mathbf{x}_{m-l}(t)+z_{m}(t),
$$

where $\mathbf{x}_{m}(t)$ is the $K \times 1$ (complex) vector of signals transmitted by the $K$ MSs in the $m$ th cell, the $K \times 1$ vector $\mathbf{h}_{m, l}(t)$ contains the channel gains $\left\{h_{m, l, k}\right\}$ towards the $m$ th BS from mobiles in the $l$ th cell (see Fig. 1 for an illustration) and $z_{m}(t)$ is complex symmetric Gaussian noise with unit power and uncorrelated over $m$ and time. We assume equal per-user power constraints

$$
\frac{1}{n} \sum_{t=1}^{n}\left|\left[\mathbf{x}_{m}(t)\right]_{k}\right|^{2} \leq \frac{P}{K}
$$

for all $m \in[1, M]$ and $k \in[1, K]$, so that the per-cell power constraint is given by $P$. Notice that model (3) assumes full frame and symbol-level synchronization among cells and users, even though extensions of the available results to the asynchronous case may be possible following, e.g., [22].

The model (3) discussed above reduces to the following special cases that will be referred to throughout this section:

- Gaussian Wyner model: This corresponds to a static scenario with symmetric inter-cell interference and cellhomogeneous channel gains, i.e., we have $L_{\ell}=L_{r}=L$ and $\mathbf{h}_{m, m-k}(t)=\alpha_{k} \mathbf{1}_{K}$ with $\alpha_{k}=\alpha_{-k}$ and $\alpha_{0}=1$. By cell-homogeneous, we mean that the channel gains do not depend on the cell index $m$, but only on the distance between interfering cells (see also discussion below on edge effects). Parameter $L$ can be referred to as the intercell interference span. Moreover, inter-cell gains $\alpha_{k} \geq 0$, $k \in[1, L]$, are deterministic (no fading) and generally known to all terminals. It is remarked that in this class of models, all users in the same cell share the same path loss. We also emphasize that the original model in [8], [9] had $L=1$, so that the system referred to here as Gaussian Wyner model is to be seen as an extension of [8], [9];

- Gaussian soft-handoff model: This corresponds to a static cell-homogeneous system like the Gaussian Wyner 
model, in which, however, there is no symmetry in the inter-cell channel gains. Specifically, we have inter-cell interference only from the left cells as $L_{\ell}=L, L_{r}=0$ and $\mathbf{h}_{m, m-k}(t)=\alpha_{k} \mathbf{1}$, with $\alpha_{0}=1$, where, as above, $\alpha_{k} \geq 0$ are deterministic quantities. This model accounts for a scenario in which users are placed at the border of the cell so that inter-cell interference is relevant only on one side of the given cell. In a number of works, including [23], [24], the Gaussian soft-handoff model is studied with $L=1$, which can be seen as describing a soft-handoff situation between two adjacent cells;

- Fading Wyner model: This model incorporates fading, accounted for by random channel gains $\mathbf{h}_{m, k}(t)$, in the Gaussian Wyner model. In particular, we have $L_{\ell}=$ $L_{r}=L$ and $\mathbf{h}_{m, m-k}(t)=\alpha_{k} \tilde{\mathbf{h}}_{m, m-k}(t)$ where vectors $\tilde{\mathbf{h}}_{m, m-k}(t), t \in[1, n]$, are independent over $m$ and $k$ and distributed according to a joint distribution $\pi_{k}$ with the power of each entry of $\tilde{\mathbf{h}}_{m, m-k}(t)$ normalized to one. For simplicity, similar to the Gaussian Wyner model, statistical symmetric inter-cell interference is assumed, i.e., $\alpha_{k}=\alpha_{-k}$ (and $\alpha_{0}=1$ ) and $\pi_{k}=\pi_{-k}$. As for temporal variations, two scenarios are typical: (i) Quasistatic fading: Channels $\tilde{\mathbf{h}}_{m, m-k}(t)$ are constant over the transmission of a given codeword (i.e., for $t \in[1, n]$ ); (ii) Ergodic fading: Channels $\tilde{\mathbf{h}}_{m, m-k}(t)$ vary in an ergodic fashion along the symbols of the codeword. The ergodic model was studied in [25] with $L=1$;

- Fading soft-handoff model: This model is the fading counterpart of the Gaussian soft-handoff model, and has $L_{1}=L, L_{2}=0$, and $\mathbf{h}_{m, m-k}(t)=\alpha_{k} \tilde{\mathbf{h}}_{m, m-k}(t)$ where $\tilde{\mathbf{h}}_{m, m-k}(t)$ are independent and modelled as for the fading Wyner model. This scenario was considered in [26], [27] (under more general conditions on the joint distribution of vectors $\left.\tilde{\mathbf{h}}_{m, m-k}(t)\right)$.

In order to remove edge effects, we will focus on the regime of a large number of cells, i.e., $M \rightarrow \infty$. This way, all cells see exactly the same inter-cell interference scenario, possibly in a statistical sense, as discussed above. An alternative approach, considered, e.g., in [8], [23], would be to consider a system in which cells are placed on a circle, which would exhibit homogeneous inter-cell interference for any finite $M$. It is noted that, however, the two models coincide in the limit of large $M$ and, in practice, results for the two models are very close for relatively small values of $M$ [21].

We now rewrite model (3) in a more compact matricial form. We drop dependence on time $t$ for simplicity. To proceed, construct a $M \times M K$ channel matrix $\mathbf{H}$ such that $m$ th row collects all channel gains to $m$ th BS, i.e., $\left[\mathbf{h}_{m, 1}^{T}\right.$, $\left.\mathbf{h}_{m, 2}^{T}, \ldots, \mathbf{h}_{m, m}^{T}, \mathbf{h}_{m, m+1}^{T}, \ldots, \mathbf{h}_{m, M}^{T}\right]$, where $\mathbf{h}_{m, m-k}^{T}$ with $k \notin\left[-L_{r}, L_{\ell}\right]$ are to be considered as zero. We can then write the $M \times 1$ vector of received signals $\mathbf{y}=\left[y_{1}, \ldots, y_{M}\right]^{T}$ as

$$
\mathbf{y}=\mathbf{H x}+\mathbf{z},
$$

where $\mathbf{x}=\left[\mathbf{x}_{1}^{T} \cdots \mathbf{x}_{M}^{T}\right]^{T}$ is the vector of transmitted signals and $\mathbf{z}$ the uncorrelated vector of unit-power Gaussian noises. From the definition above, it is clear that, in general, $\mathbf{H}$ is a finite-band matrix (in the sense that only a finite number of diagonals have non-zero entries). Moreover, it is not difficult

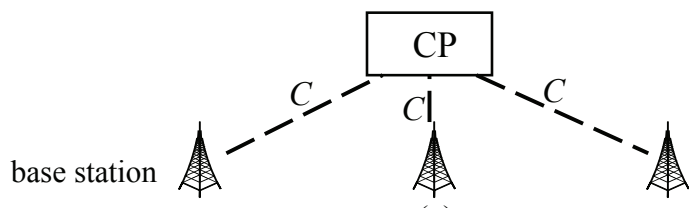

(a)

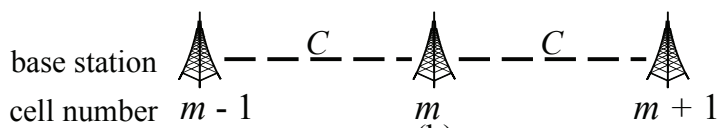

(b)

Fig. 4. Backhaul models for MCP: (a) Central processor (CP) with finitecapacity backhaul links (of capacity $C$ ); (b) Local finite-capacity backhaul between adjacent BSs (of capacity $C$, uni- or bi-directional). Dashed lines represent backhaul links.

to see that for Gaussian Wyner and Gaussian soft-handoff models, matrix $\mathbf{H}$ has a block-Toeplitz structure, which will be useful in the following.

2) Downlink: Define as $\mathbf{y}_{m}$ the $K \times 1$ vector of signals received by the $K \mathbf{M S s}$ in the $m$ th cell, $\mathbf{y}=\left[\mathbf{y}_{1}^{T} \cdots \mathbf{y}_{M}^{T}\right]^{T}$, and $\mathrm{x}$ as the $M \times 1$ transmitted signal by the BSs. We then have from (2)

$$
\mathbf{y}=\mathbf{H}^{\dagger} \mathbf{x}+\mathbf{z}
$$

where $\mathbf{z}$ is the vector of unit-power uncorrelated complex Gaussian noise and channel matrix $\mathbf{H}$ is defined as above. We assume a per-BS (and thus per-cell) power constraint $\frac{1}{n} \sum_{t=1}^{n}\left|[\mathbf{x}(t)]_{m}\right|^{2} \leq P$ for all $m \in[1, M]$.

3) Multi-Cell Processing: For both uplink and downlink, we will consider the two following models for the backhaul links that enable MCP, see Fig. 4.

- Central processor (CP) with finite-capacity backhaul (Fig. 4-(a)): In this case, all BSs are connected to a CP for joint decoding (for uplink) or encoding (for downlink) via finite-capacity backhaul links of capacity $C$ [bits/channel use]. Recall that the original works [8], [9], [12] assume unlimited backhaul capacity, i.e., $C \rightarrow \infty$;

- Local finite-capacity backhaul between adjacent BSs (Fig. 4-(b)): Here BSs are connected to their neighboring BSs only via finite-capacity links of capacity $C$ [bits/channel use], that may be uni- or bi-directional.

It is noted that two models coincide in the case of unlimited backhaul capacity $C \rightarrow \infty$. Also, we remark that another popular model assumes that only BSs within a certain cluster of cells are connected to a CP for decoding. This model will be considered as well, albeit briefly, below.

\section{Capacity Results for the Wyner Uplink Model}

In the rest of this section, we elaborate on the per-cell sum-rate achievable for the uplink of the Wyner-type models without relays reviewed above. When not stated otherwise, we will focus on the Gaussian Wyner model. Fading models are discussed in Sec. III-C5. Throughout, we assume that channel state information (CSI) on gains $\left\{\alpha_{k}\right\}$ is available at all nodes. 


$$
R_{S C P}(P, F)= \begin{cases}\frac{1}{F} \log _{2}\left(1+\frac{F P}{1+2 F P \sum_{k=1}^{[L / F]} \alpha_{k F}^{2}}\right) & \text { if } F \leq L \\ \frac{1}{F} \log _{2}(1+F P) & \text { if } F>L\end{cases}
$$

1) Single-Cell Processing (SCP) and Spatial Reuse: Consider at first a baseline scheme, where Single-Cell Processing (SCP) is performed, so that each BS decodes individually its own $K$ users by treating users in other cells as Gaussian noise. A standard technique to cope with inter-cell interference is spatial reuse, that consists in activating at a given time (or equivalently in a given subband) only one cell every $F \geq 1$ cells. Parameter $F$ is referred to as the spatial reuse factor. SCP with special reuse is easily seen to achieve the per-cell sum-rate in Eq. 7 where $L$ is the inter-cell interference span. Rate (7) is obtained by either letting all users in a given cell transmit at the same time with power $F P / K$, which we refer to as Wide-Band (WB) transmission, or by intra-cell TDMA, whereby each user in a cell transmits with power $F P$ for a fraction of time $1 / K$. Notice that such power allocations satisfy the per-block power constraint (4), due to the fact that each cell transmits for a fraction $1 / F$ of the time.

A few remarks are in order. First, as seen in (7), if the reuse factor $F$ is larger than the inter-cell interference span $L$, SCP with spatial reuse completely eliminates inter-cell interference and provides a non-interference-limited behavior with per-cell multiplexing gain ${ }^{2}$ equal to $1 / F$, whereas otherwise the system operates in the interference-limited regime [8], [9]. From this, we conclude that the presence of inter-cell interference, if handled via SCP, leads to a rate degradation with respect to an interference-free system at high SNR given by a factor of $L$ [8]. In the low SNR regime, instead, where noise dominates inter-cell interference, using the formalism of $[28]^{3}$, it can be seen that inter-cell interference does not cause any increase in the minimum (transmit) energy-per-bit necessary for reliable communications $E_{b} / N_{0 \mathrm{~min}}$, which equals $\ln 2=-1.59 \mathrm{~dB}$, as for interference-free channels. However, if one observes also the slope of the spectral efficiency $S_{0}[\mathrm{bits} / \mathrm{s} / \mathrm{Hz} /(3 \mathrm{~dB})]$, which accounts for a higher-order expansion of the spectral efficiency as the SNR $P \rightarrow 0$, the loss due to inter-cell interference is seen also in the low-SNR regime. In fact, we have for rate (7):

$$
\frac{E_{b}}{N_{0 \text { min }}}=\ln 2 \text { and } S_{0}= \begin{cases}\frac{2}{F\left(1+4 \sum_{k=1}^{L L / F\rfloor} \alpha_{k F}^{2}\right)} & \text { if } F \leq L \\ \frac{2}{F} & \text { if } F>L\end{cases}
$$

where we recall that interference-free channels have $S_{0}=2$. The conclusions here are related to the analysis in [29] on the suboptimality of TDMA for multiuser channels. As shown

\footnotetext{
${ }^{2}$ The per-cell multiplexing gain is defined as the limit $\lim _{P \rightarrow \infty} R(P) / \log P$, where $R(P)$ is the given achievable per-cell sum-rate. A system is said to be interference-limited if the multiplexing gain is zero, and non-interference-limited otherwise.

${ }^{3}$ Reference [28] proposes to expand an achievable rate $R$ as a function of the energy-per-bit $E_{b}=P / R$ as $R \simeq \frac{S_{0}}{3 d B}\left(\frac{E_{b}}{N_{0}} d B-\frac{E_{b}}{N_{0}} \min , d B\right)$, where $N_{0}$ is the noise spectral density (normalized to 1 here) and $\frac{E_{b}}{N_{0}}$ min $=$ $\frac{1}{R(0)}$ and $S_{0}=(2 \ln 2) \frac{(\dot{R}(0))^{2}}{(-R(0))}$, where $R(P)$ is the considered rate (in bits/channel use) as a function of the power $P$.
}

below, MCP allows to overcome the limitations of SCP and spatial reuse discussed here.

2) Unlimited Backhaul: Assume at first unlimited backhaul links to a $\mathrm{CP}$, i.e., $C \rightarrow \infty$. The per-cell sum-capacity $R_{M C P}(P)$ with $\mathrm{MCP}$ in this scenario (for any $M$ ) is given by [9]:

$$
\begin{aligned}
R_{M C P}(P) & =\frac{1}{M} \log _{2} \operatorname{det}\left(\mathbf{I}_{M}+\frac{P}{K} \mathbf{H H}^{\dagger}\right) \\
& =\frac{1}{M} \sum_{m=1}^{M} \log _{2}\left(1+\frac{P}{K} \lambda_{i}\left(\mathbf{H H}^{\dagger}\right)\right) \\
& =\int_{0}^{\infty} \log _{2}\left(1+\frac{P}{K} x\right) d F_{\mathbf{H}} \mathbf{H}^{\dagger}(x),
\end{aligned}
$$

where $\lambda_{i}\left(\mathbf{H H}^{\dagger}\right)$ denotes eigenvalues of the argument matrix and $F_{\mathbf{H H}^{\dagger}}(x)$ is the empirical distribution of such eigenvalues:

$$
F_{\mathbf{H H}^{\dagger}}(x)=\frac{1}{M} \sum_{m=1}^{M} 1\left(\lambda_{i}\left(\mathbf{H H}^{\dagger}\right) \leq x\right) .
$$

The per-cell capacity (9) is achieved by performing ideal multi-user detection at the $\mathrm{CP}$ (which can in practice be realized by following approaches such as [30]). Moreover, it can be attained via both an intra-cell TDMA scheme where users transmit with power $P$ for a fraction of time $1 / K$ and by the WB scheme (whereby all users transmit with full power $P / K$ at all times). It is noted that the optimality of TDMA is strictly dependent on the per-block power constraint (4), and would not hold under more restrictive conditions, such as peak or per-symbol power constraints. More general conditions under which TDMA is optimal, under per-block power constraints, can be found in [8]. For instance, from [8], it is found that TDMA would generally not be optimal in scenarios where users had different intra- and inter-cell channel gains, such as in fading scenarios (see Sec. III-C5). For the Gaussian Wyner model of interest here, using Szego's theorem, we get that for $M \rightarrow \infty$ rate (9) can be written [9] in a simple integral form as in (11). Expression (11) can be interpreted by considering the case $K=1$ (without loss of generality, given the optimality of intra-cell TDMA) and identifying the signal received at the $\mathrm{CP}$ as the output (for each time instant) of a Linear Time Invariant (LTI) filter, whose input is given by the signals transmitted by the MSs and whose impulse response is $\delta_{m}+\sum_{k=1}^{L} \alpha_{k} \delta_{m-k}+\sum_{k=1}^{L} \alpha_{k} \delta_{m+k}\left(\delta_{m}\right.$ is the Kronecker delta). This integral cannot be evaluated in closed form in general. It should be noted that in other scenarios, such as the Gaussian soft-handoff model with $L=1$, the corresponding integral can be instead calculated in closed form [23][24]. Notice that multiplexing gain of the MCP capacity (11) is one, as for an interference-free scenario. Moreover, the minimum energy-per-bit is given by

$$
{\frac{E_{b}}{N_{0 \text { min }}}}=\frac{\ln 2}{\left(1+2 \sum_{k=1}^{L} \alpha_{k}^{2}\right)},
$$




$$
R_{M C P}(P)=\int_{0}^{1} \log _{2}\left(1+P\left(1+2 \sum_{k=1}^{L} \alpha_{k} \cos (2 \pi k \theta)\right)^{2}\right) d \theta
$$

showing an energy gain due to $\mathrm{MCP}$ with respect to SCP and to an interference-free system given by $\left(1+2 \sum_{k=1}^{L} \alpha_{k}^{2}\right)$ (parameter $S_{0}$ is not reported here for lack of space but can be obtained from [21]).

3) Limited Backhaul to the CP: Consider now the scenario in Fig. 4-(a), where the BSs are connected to a $\mathrm{CP}$ via finitecapacity links. At first, we remark that the achievable per-cell sum-rate is limited by the cut-set upper bound

$$
R_{U B}(P, C)=\min \left\{C, R_{M C P}(P)\right\} .
$$

Moreover, here, the performance depends on the knowledge of codebooks used by the MSs at the BSs. Assume at first that the BSs are unaware of the codebooks used by the MSs (oblivious BSs). In [31], this scenario is considered, and a per-cell achievable sum-rate is derived for a strategy whereby the BSs simply compress (to $C$ bits/channel use) and forward the received signals to the CP. Compression is followed by (random) binning, exploiting the fact that the other BSs have correlated information, according to standard techniques in distributed source coding (see, e.g., [32]). Decoding at the $\mathrm{CP}$ is done by jointly ${ }^{4}$ decompressing the signals forwarded by the BSs and decoding the codewords transmitted by the users. A simple expression is found for this achievable rate in [31]:

$$
R_{O B L}(P, C)=R_{M C P}\left(P\left(1-2^{-r}\right)\right),
$$

where $R_{M C P}$ is defined in (11) and $r$ is the solution of the fixed-point equation:

$$
R_{O B L}\left(P\left(1-2^{-r}\right)\right)=C-r .
$$

In other words, the finite-capacity links entail a SNR loss of $\left(1-2^{-r}\right)$ with respect to the unlimited backhaul capacity (11). It is noted that parameter $r$ has the interpretation of the amount of capacity $C$ that is wasted to forward channel noise to the CP [31], [32]. Also, we remark that rate (14) does not match the upper bound (13) in general. However, this is not always the case, and thus optimality of (14) is proved, in the regimes with $C \rightarrow \infty$ (in which compression noise becomes negligible), on the one hand, and $P \rightarrow \infty$ (in which the performance is limited by $C$ ), on the other. It is also interesting to point out that for low-SNR, the power loss of the oblivious scheme at hand with respect to (11) is quantified by calculating parameter $E_{b} / N_{0 \min }$ as $E_{b} / N_{0 \min }=$ $\ln 2\left(1+2 \sum_{k=1}^{L} \alpha_{k}^{2}\right)^{-1}\left(1-2^{-C}\right)$. Comparing this with (12), one sees that in the low-SNR regime, the loss of (14) with respect to (11) is neatly quantified by $1-2^{-C}$. As a final remark, the optimal multiplexing gain of one is achieved if the backhaul capacity $C$ scales as $\log P$, which coincides with the optimal behavior predicted by the upper bound (13).

We now consider a different scenario where BSs are informed about the codebooks used by the MSs both in the

\footnotetext{
${ }^{4}$ It is interesting to notice that while joint decompression/ decoding yields no performance benefits for regular interference-free systems [33], this is not the case in the presence of interference (see also [34], [35]).
}

same cell and in the interfering cells. In [31], a scheme is considered where partial decoding is carried out at the BSs. According to this approach, each MS splits its message and transmitted power into two parts: The first is intended to be decoded locally by the in-cell BS (with possible joint decoding also of the signals from the interfering BSs) and transmitted over the limited link to the CP, while the second part is processed according to the oblivious scheme and is decoded by the CP as discussed above. This scheme is shown to provide advantages over the oblivious rate (14) when the inter-cell interference is low (it is easy to see that it is optimal for $\alpha_{k}=0, k>0$ ) and for small $C$. Another strategy that exploits codebook knowledge at the BSs is the structured coding scheme proposed in [36] and reviewed below.

In [36], it is proposed that the BSs, rather than decoding the individual messages (or parts thereof) of the MSs as in [31], decode instead a function of such messages or, more precisely, of the corresponding transmitted codewords. The key idea that enables this operation is the use of structured, rather than randomly constructed, codes. Each MS employs the same nested lattice code and the signal received at any $m$ th BS can be written from (11) as $y_{m}=\sum_{k=-L}^{L} \alpha_{k} x_{m-k}+z_{m}$. Recalling that a lattice code is a discrete group, the (modulo ${ }^{5}$ ) sum of the lattice codewords $x_{m-k}$, weighted by integer coefficients, is still a codeword in the same lattice code and can thus be decoded by the $m$ th $\mathrm{BS}$. The problem is that the channel coefficients $\alpha_{k}$ are generally not integers. The $m$ th BS can however decode an arbitrary linear combination $\sum_{k=-L}^{L} b_{k} x_{m-k}$ with $b_{k} \in \mathbb{Z}$ (and by symmetry $b_{k}=b_{-k}$ ) and $b_{0} \neq 0$ and treat the remaining part of the signal as Gaussian noise. The index of the decoded codeword can then be sent to the $\mathrm{CP}$, that decodes based on all received linear combinations. This leads to the achievable rate [36] shown in (16) where $\mathcal{B}=\left\{\left(b_{0}, . ., b_{L}\right) \in \mathbb{Z}: b_{0} \neq 0\right.$ and $\left.b_{0}^{2}+2 \sum_{k=1}^{L} b_{k}^{2} \leq 1+P\left(1+2 \sum_{k=1}^{L} \alpha_{k}^{2}\right)\right\}$. As shown in [36], this rate may outperform (14) for low and high intercell interference ([36] considers the case $L=1$ ). Moreover, [36] proves that rate (16) can be improved by superimposing additional messages to the lattice codewords.

4) Local BS backhaul: In this section, we turn to the model in Fig. 4-(b), where BSs are connected only to their neighboring BS via finite-capacity links. At first, for reference, we consider the related cluster-decoding setting of [37], where each BS, say the $m$ th, can decode based not only on the locally received signal $y_{m}$ but also on the received signals from $i_{\ell}$ BSs on the left $\left(y_{m-k}\right.$ with $\left.k \in\left[1, i_{\ell}\right]\right)$ and $i_{r}$ BSs on the right $\left(y_{m+k}\right.$ with $\left.k \in\left[1, i_{r}\right]\right)$. Notice that this accounts for a situation where unlimited capacity backhaul links connect BSs, but only within a certain range of cells. Reference [37] obtains the maximum multiplexing gain of this setting for a Gaussian soft-handoff model with $L=1$ with intra-cell

\footnotetext{
${ }^{5}$ The modulo operation is taken with respect to the coarse lattice forming the nested lattice code.
} 


$$
R_{L A T}=\min \left\{C, \max _{\left(b_{0}, . ., b_{L}\right) \in \mathcal{B}}-\log \left(b_{0}^{2}+2 \sum_{k=1}^{L} b_{k}^{2}-\frac{P\left(b_{0}+2 \sum_{k=1}^{L} \alpha_{k} b_{k}\right)^{2}}{1+P\left(1+2 \sum_{k=1}^{L} \alpha_{k}^{2}\right)}\right)\right\}
$$

TDMA (or equivalently $K=1$ ). The model of [37] also assumes that MSs are aware, before choosing the transmitted codewords, of the messages of the MSs in $J_{\ell}$ cells on the left and $J_{r}$ cells on the right. This is a simple way to account for cooperation at the MS level, and will be further discussed in Sec. III-E. The maximum multiplexing gain is given by

$$
\frac{J_{\ell}+J_{r}+i_{\ell}+i_{r}+1}{J_{\ell}+J_{r}+i_{\ell}+i_{r}+2}
$$

showing that with clustered decoding the multiplexing gain is generally less than one, but larger than $1 / 2$, as achievable with SCP and spatial reuse (see Sec. III-C1). Moreover, this shows that (for the soft-handoff model), left and right side informations have the same impact on the multiplexing gain, and the same applies to cooperation at the MSs or cluster decoding. Multiplexing gain (17) is achieved by successive interference cancellation at the BSs, where BSs exchange information about the decoded signals (see also below), and Dirty Paper Coding (DPC)-based cooperation at the users. It is noted that this scheme requires knowledge of the codebooks used in adjacent cells by both BSs and MSs. A model with cluster decoding at the BSs, but no cooperation amongst the mobiles, is considered in [38, Section IV], where similar general conclusions about the multiplexing gain are obtained.

In the presence of finite-capacity backhaul, the inter-BS links can be used to provide limited-rate information about the received signal or a processed version thereof to adjacent BSs. Such "relaying" has in general the double purpose of providing information about the useful signal of the recipient but also of the interference. This observation has also been made in the context of interference relay channels (see review in [39]). Along these lines, it is noted that the model and techniques at hand are very related to interference channels with "conferencing" decoders studied in [34], [40]. Consider, as in [41], a soft-handoff model with $L=1$ and unidirectional backhaul links allowing information to be passed to the right. Assuming knowledge of only the local codebook, a successive decoding scheme can be devised in which each BS decodes the local message and sends the quantized decoded codeword to the neighboring (right) BS for interference mitigation. It is not difficult to see that such a scheme has zero multiplexing gain since it is not able to fully mitigate the interference. This is in contrast with the case where BSs have information about the codebooks used in adjacent cells. In this case, as in [37] (see discussion above), it becomes possible to perform joint decoding of the local message and of (possibly part of) the interfering message, and to use the backhaul link to convey directly hard information (messages) rather than soft information about the decoded codewords. This allows a non-interference limited behavior to be attained: Specifically, assuming that $C$ grows like $\beta \log P$, the multiplexing gain $\min (1,0.5+\beta)$ can be attained [41].

5) Fading Channels: In this section, we discuss available results on the sum-rate of fading Wyner and soft-handoff models. We consider both quasi-static and ergodic fading below.

a) Quasi-static Fading: With quasi-static fading, the outage capacity is typically used as a performance criterion [42]. This is, generally speaking, the maximum rate that guarantees reliable transmission for a given percentage of channel realizations (the measure of whose complement is referred to as outage probability). This setting implies either lack of CSI at the users (so that rate adaptation is not possible) or inelastic constant-rate applications. Using such a criterion in a largescale cellular system with MCP proves to be challenging: In fact, on the one hand, defining outage as the event where any of the users' messages are not correctly decoded leads to uninteresting results as the number of cells $M$ grows large; On the other hand, defining individual outage events, as studied in [43] for a two-user MAC, appears to be analytically intractable for large systems (see [44] for related work).

A tractable performance criterion is instead obtained by considering the achievable per-cell sum-rate (9) for given channel realizations in the limit as the number of users per cell $K$ and/or the number of cells $M$ grow large, where the limit is defined in an almost sure sense. It is noted that such per-cell sum-rate is achievable by appropriate choice of distinct rates by the MSs, and such choice depends on the current realization of the channel matrices. The practical significance of this criterion is thus limited to instances in which, thanks to appropriate signaling, such rate adaptation is possible. Therefore, we review these results below as they are practically more relevant in the context of ergodic channels.

b) Ergodic Fading: With ergodic fading, the per-cell sum-capacity is given by the expectation of $(9 \mathrm{c})$ with respect to the distribution of $\mathbf{H}$, i.e., $R_{M C P}^{\text {erg }}(P)=\mathbb{E}\left[R_{M C P}(P)\right]$. It is noted that such rate can be attained, due to the (stochastic) symmetry of the considered model (neglecting edge effects by taking the limit $M \rightarrow \infty$ ) by equal rate allocation to all users. Moreover, it is achieved by a WB scheme (all users transmit at the same time), the rate of intra-cell TDMA being generally smaller. This is in contrast with Gaussian (unfaded) models, as discussed in Sec. III-C1, and is in line with standard results for multiple access channels [45], [46]. It is also noted that with SCP, when treating interference as noise as in (7), intra-cell TDMA may instead be advantageous over WB when intercell interference takes place and exceeds a given threshold [46]. Some performance comparison between intra-cell TDMA and spatial reuse in the presence of MCP for the soft-handoff model with $L=1$ and Rayleigh fading can be found in [47].

To evaluate $R_{M C P}^{e r g}(P)$, one can either use approximations based on bounding techniques as in [25] or the fact that, if $F_{\mathbf{H H}^{\dagger}}(x)$ converges almost surely in some asymptotic regime of interest to some limiting distribution (spectrum) $F(x)$, then $R_{M C P}^{e r g}(P)$ converges to (9c) with $F_{\mathbf{H H}^{\dagger}}(x)=F(x)$ (see, e.g., [48]). We discuss below two such regimes.

Consider first the asymptotics with respect to $K$ and $M$ (with the inter-cell interference span $L$ kept fixed). Let us 


$$
R_{M C P}^{e r g}(P)=\int_{0}^{1} \log _{2}\left(\begin{array}{c}
1+P\left(1-\mu^{2}\right)\left(1+2 \sum_{k=1}^{L} \alpha_{k}^{2}\right) \\
+P \mu^{2}\left(1+2 \sum_{k=1}^{L} \alpha_{k} \cos (2 \pi k \theta)\right)^{2}
\end{array}\right) d \theta
$$

assume that the distribution $\pi_{k}$ of vectors $\tilde{\mathbf{h}}_{m, k}$ (recall Sec. III-B) is such that each channel vector can be seen as a realization of a stationary and ergodic process with unit power and mean $0 \leq \mu \leq 1$ (and thus variance $1-\mu^{2}$ ). In this case, it can be verified that matrix $\mathbf{H H}^{\dagger}$ converges almost surely to a deterministic Toeplitz matrix due to the strong law of large numbers [8]. Now, using Szego's theorem, similarly to (11), we have that for $K \rightarrow \infty$ and $M \rightarrow \infty$ (taken in this order) we obtain (18) (see [25]).

Notice that we recover (11) for $\mu=1$, which corresponds to an unfaded scenario. It can be proved, similarly to [25], that (18) is decreasing in $\mu^{2}$, which implies that fading is beneficial in the limit of a large number of users. It is remarked that this may not be the case for a finite number of users $K$, as can easily be seen by noticing that for $K=1$ and no inter-cell interference, one obtains a point-to-point link for which fading is known not to increase the rate [25], [26]. It is noted that the potential benefits of fading are related to the independence of the fading gains towards different BSs and thus cannot be mimicked by the MSs [25][23] (see also [8, Section 5.1.2] for a discussion on the effect of fading on the multiplexing gain, and on the power offset term). Moreover, from Jensen's inequality, it can be seen that (18) is an upper bound on the ergodic per-cell capacity for any number of users $K$ [25]. Finally, rate (18) does not depend on the actual (stationary and ergodic) distribution $\pi_{k}$ but only on its first two moments.

Consider now a regime where $K$ is fixed and $M$ grows to infinity. An approximation of the limiting spectrum $F(x)$, and thus of the per-cell rate $R_{M C P}^{e r g}(P)$, has been obtained in [49] for the fading Wyner model with $L=1$ and Rayleigh fading using free probability tools. Such approximation is seen to be accurate only for small values of the interference gain $\alpha_{1}$. In [26], [27], exact results on the convergence of per-cell rate (9a) are studied for the fading soft-handoff model. Almost sure convergence to a limit that depends on the Lyapunov exponent of a certain product of matrices is shown (see also [50] for related work). A central limit theorem is also proven in [27] along with a corresponding large deviation result, providing evidence to the fact that, given the limited randomness present in matrix $\mathbf{H}$ (due to the banded structure), convergence is slower than in classical random matrix theory (see, e.g., [51]). Finally, [52] characterizes the high-SNR behavior (in the sense of [53]) of (9a) as $M$ grows large and $K=1$ user and $L=1$. Performance bounds are also provided for $K>1$. The result shows that such behavior depends on the specific distribution $\pi_{k}$, lending evidence to the conclusion that, in the case of finite-band matrices, the limit spectrum depends on the entries' distribution, unlike standard random matrix theory [25], [48]. We also remark that in the fading soft-handoff model with Rayleigh fading, $L=1$, and intra-cell TDMA (or equivalently $K=1$ ), the ergodic rate $R_{M C P}^{e r g}(P)$ can be found in a compact integral form as shown in [54], [23]. Reference [47] obtains related bounds for $K>1$.
Finally, we point to [46], where the effect of fading on a Wyner model with ideal cooperation only between adjacent cell sites is studied.

c) MIMO Fading Models: Another extension is to consider multiple antennas at the BS, with fading from each antenna to each user. Uplink models comparing SCP to $\mathrm{MCP}$ in this context are considered in [55], [56]. In [55] an asymptotic regime is considered in which the number of antennas at the base station, and the number of mobiles, grow large together, in a circular Wyner model. It is shown that the degrees of freedom depend on the system loading (number of users per base station antenna), but, if SCP and MCP are both optimally loaded (respectively), then MCP gains over SCP by a factor of three, but the gap can be reduced to a factor of two via the use of a re-use factor of two, with even and odd cells in separate bands.

d) Channel uncertainty: Channel uncertainty has not been adequately treated in the network MIMO literature to date. Its importance can be seen from the point to point MIMO channel where it is known that the number of transmit antennas should not exceed the number of symbols in the coherence block [57]. The reason is that part of the block of symbols must be used for training so that the MIMO channels can be measured at the receiver. If the coherence time is long relative to the symbol period then the number of antennas can be large, but if the coherence time is one symbol duration then one antenna is optimal. Note that the limiting asymptotics discussed in c) implicitly assume that the coherence time is growing with the number of users. Thus, channel uncertainty has implications for MIMO scalability, and we will discuss this issue further in Section V.

6) Numerical Results: We now focus on a numerical example for a Gaussian Wyner model with $L=1$. Fig. 5 shows the per-cell sum-rate achievable by the techniques discussed above, namely SCP with spatial reuse $F=1$ and $F=2$ (7), ideal MCP (11), oblivious processing at the BSs (14) with backhaul capacity $C=5$ and lattice coding (16) with $C=5$. We have $P=15 d B$ and the inter-cell interference power gain $\alpha_{1}^{2}$ is varied. It can be seen that SCP with spatial reuse $F=1$ provides interference-limited performance, while with $F=2$ inter-cell interference is eliminated, but at the cost of possibly reducing the achievable rate. MCP provides remarkable performance gains, and can potentially benefit from larger inter-cell power gains $\alpha_{1}^{2}$. When the backhaul capacity is restricted to $C=5$ (which is of the order of the per-cell achievable rates at hand), it is seen that by choosing the best between the oblivious BS scheme and the latticebased scheme, one performs fairly close to the bound of ideal MCP. Moreover, lattice-based coding has performance advantages over oblivious processing for sufficiently large or small interference, large $P$ (not shown here) and moderate $C$. Increasing the capacity $C$ to say $C=8$, leads to an almost ideal rate with the oblivious strategy (consistently with its 


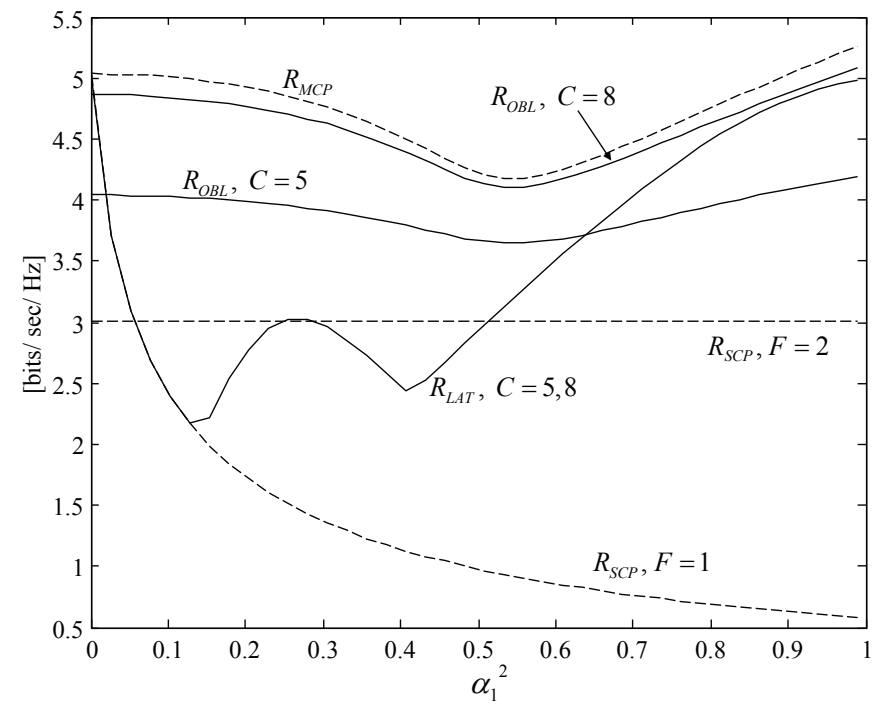

Fig. 5. Uplink of a Gaussian Wyner model with $L=1$ : Per-cell sum-rate achievable by SCP with spatial reuse $F=1$ and $F=2$ (7), ideal MCP (11), oblivious processing at the BSs (14) with backhaul capacity $C=5$ and lattice coding (16) with $C=5$ versus the inter-cell interference power gain $\alpha_{1}^{2}(P=15 d B)$.

asymptotic optimality for $P \rightarrow \infty$ ), while lattice coding does not improve its performance.

\section{Capacity Results for the Wyner Downlink Model}

In this section, we review corresponding results for the downlink. Reference results using SCP and frequency reuse are similar to Sec. III-C1 and need not be discussed here. We focus, as for the uplink, on Gaussian models and briefly discuss the impact of fading in Sec. III-D3.

1) Unlimited Backhaul: Consider first the case of unlimited backhaul. Reference [12] derives achievable rates based on the linear precoding dirty paper coding strategy of [58]. The percell sum-capacity is instead derived in [23] using the uplinkdownlink duality results of [59] as

$$
R_{M C P}(P)=\frac{1}{M} \min _{\boldsymbol{\Lambda}} \max _{\mathbf{D}} \log _{2} \frac{\operatorname{det}\left(\boldsymbol{\Lambda}+\frac{P}{K} \mathbf{H D H} \mathbf{H}^{\dagger}\right)}{\operatorname{det}(\boldsymbol{\Lambda})},
$$

with $\boldsymbol{\Lambda}$ and $\mathbf{D}$ being diagonal $M K \times M K$ matrices with the constraints $\operatorname{tr}(\boldsymbol{\Lambda}) \leq M$ and $\operatorname{tr}(\mathbf{D}) \leq M$. This rate is known to be achieved by dirty paper coding at the CP. For the Gaussian Wyner (circulant) model, it can be shown that the per-cell sumcapacity (19) is exactly equal to the corresponding capacity for the uplink (11) for $M \rightarrow \infty$. It follows that, as for the uplink, intra-cell TDMA, where only one user is served per-cell, is optimal with Gaussian (unfaded) channels.

2) Limited Backhaul to a CP: Similarly to the uplink, strategies to be used in the presence of limited backhaul to a CP depend on the level of codebook information available at the BSs. For oblivious BSs, reference [60] proposes to perform joint DPC under individual power constraint at the $\mathrm{CP}$ and then send the obtained codewords to the corresponding BSs via the backhaul links. The BSs simply transmit the compressed DPCcodewords. Since the transmitted quantization noise decreases the overall SNR seen by the MSs, joint DPC at the CP is designed to meet lower SNR values and tighter power constraints than those of the unlimited setup [23]. The resulting per-cell rate is shown to be equal to (11) but with a degraded SNR

$$
\bar{P}=\frac{P}{1+\frac{1+P\left(1+2 \sum_{k=1}^{L} \alpha_{k}^{2}\right)}{2^{C}-1}}
$$

due to quantization noise. Similarly to the corresponding result (14) for the uplink, this rate is generally suboptimal but it achieves cut-set bound (13) (which is still a valid bound also for the downlink) for $C \rightarrow \infty$ (where the compression noise is dominant). However, unlike for the uplink, this rate is not optimal for $P \rightarrow \infty$ : This fact can be understood by noticing that in the high-SNR regime, the compression noise dominates the performance, and, in the downlink, the compression noise is dealt with independently by each MS, unlike in the uplink, where decompression is performed jointly at the CP. Interestingly, for low-SNR the power loss in terms of $E_{b} / N_{0 \text { min }}$ turns out to be exactly the same as for the uplink, being given by $\left(1-2^{-C}\right)$. Moreover, as for the uplink rate (14), optimal multiplexing gain of 1 per-cell is achieved if $C \sim \log P$.

Reference [60] also considers the case where the BSs possess codebook information about adjacent BSs belonging to a given cluster and proposes to perform DPC within the given cluster. The main conclusion of [60] is that the oblivious scheme is the preferred choice for small-to-moderate SNRs or when the backhaul capacity $C$ is allowed to increase with the SNR. On the other hand, for high SNR values and fixed capacity $C$, a system with oblivious BSs is limited by the quantization noise, and knowledge of the codebooks at the BSs becomes the factor dominating the performance.

3) Fading Channels: Following the discussion in Sec. III-C5, here we focus on the ergodic fading scenario. For this setting, the per-cell sum-capacity is given by $R_{M C P}^{e r g}(P)=$ $\mathbb{E}\left[R_{M C P}(P)\right]$ using (19). Evaluating this quantity is not an easy task due to the min-max operation involved. In [23], upper and lower bounds on $R_{M C P}^{e r g}(P)$ are derived for the fading soft-handoff model with $L=1$ and Rayleigh fading, along with asymptotic SNR characterizations. An important finding from such analysis is that, for large number $K$ of users per cell, the per-cell sum-rate capacity scales as $\log \log K$, which is the same type of scaling as for interference-free systems. A suboptimal scheme is then proposed in [61] based on zero-forcing (ZF) beamforming and a simple user selection (scheduling) rule whereby one user is served in each cell at any given time in an intra-cell TDMA fashion. It is found that, even this suboptimal scheme is able to achieve the same optimal scaling law of $\log \log K$ with Rayleigh fading.

An illustration of the achievable per-cell rates in a fading Wyner model with $L=1$ and $\alpha_{1}^{2}=0.4$ is shown in Fig. 6. Specifically, the per-cell achievable rates with SCP and spectral reuse $F=1$ and $F=2$ (obtained similarly to Sec. III-C1), with ideal MCP (shown is the upper bound of [23]) and with the ZF beamforming and scheduling scheme of [61] are plotted versus the power $P$ and for $K=50$ users per cell. The interference-limited behavior of SCP with $F=1$ is apparent and so is the performance gain achievable via 


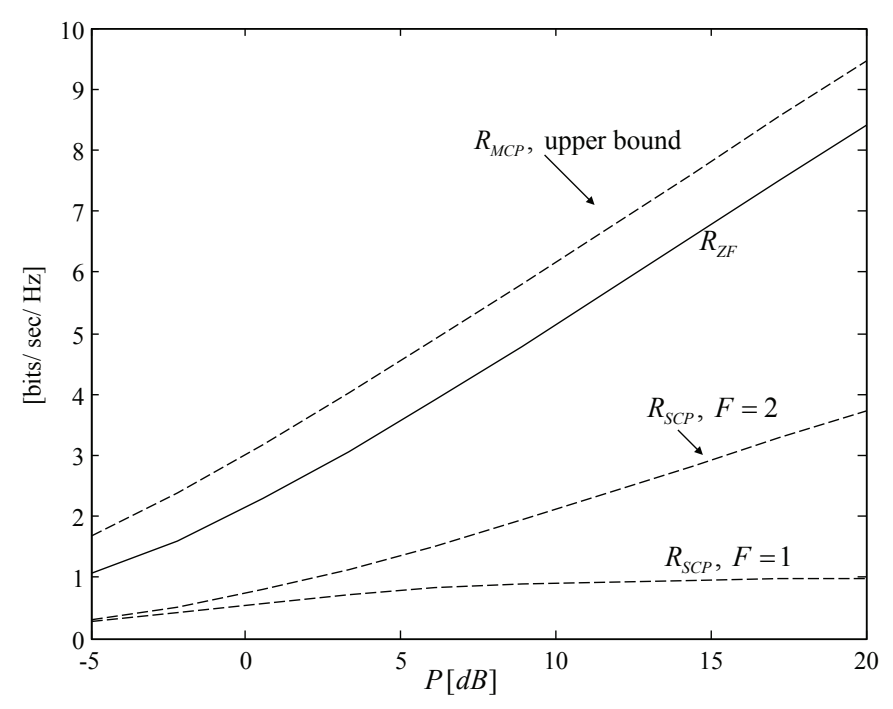

Fig. 6. Downlink of a fading Wyner model with $L=1$ : Per-cell sum-rates achievable with SCP and spectral reuse $F=1$ and $F=2$, ideal MCP, and with the $\mathrm{ZF}$ beamforming and scheduling scheme of [61] are plotted versus the power $P\left(K=50, \alpha_{1}^{2}=0.4\right)$.

MCP. It is also interesting to notice that the suboptimal ZF beamforming scheme performs relatively close to the upper bound set by ideal MCP.

\section{E. Relay-aided Models}

In modern cellular systems, the presence of dedicated relays is considered to be instrumental in extending coverage by enabling multi-hop communications or, more generally, cooperation at the MS level [62]. Here, we briefly review a model that accounts for the presence of dedicated relays, one per cell, in a Wyner-type setting (first considered in [63]). We focus on the uplink for simplicity and assume that the users are sufficiently far from the BSs so that the direct link from MS to BS can be neglected. We thus end up with two Wynertype models, one from the users to the relays and one from the relays to the BSs (see Fig. 7). We will refer to these as first and second hop, respectively. We assume that the relays are full-duplex so that they can transmit and receive at the same time. The protocols we consider, except when stated otherwise, work by pipelining transmission on the two hops: The mobiles send a new message to the relays in every block, while the relays transmit to the BSs a signal obtained by processing the samples received in the previous block. Given the assumption of no direct link between mobiles and BSs, it is easy to see that results with half-duplex relays are immediately derived by halving the spectral efficiencies obtained for the full-duplex case (a new message can only be sent once every two blocks).

Denoting as $L_{\ell}$ and $L_{r}$ the maximum inter-cell interference spans on the left and right, respectively, for the two hops, we can write the signal model, similar to (5), as follows. The $M \times 1$ signal received at the relays can be written as

$$
\mathbf{y}_{R}=\mathbf{H x}+\mathbf{T} \mathbf{x}_{R}+\mathbf{z}_{R}
$$

where $\mathbf{H}$ is defined as in (5), and contains the channel gains for the first hop (MSs-relays), $\mathbf{x}$ is as in (5) and $\mathbf{z}_{R}$ is the
Gaussian noise. The new element here is the $M \times 1$ signal $\mathbf{x}_{R}$ transmitted by the relays. The possible interference among relays in different cells is accounted for by matrix $\mathbf{T}$. Here, we assume that there is interference only between relays in adjacent cells, and that such interference is symmetric, so that $\mathbf{T}$ is a symmetric Toeplitz matrix with first row equal to $[0$ $\left.\mu \mathbf{0}_{M-2}^{T}\right]$, where $\mu$ represent the inter-relay gains. Finally, the signal received at the BSs is given by

$$
\mathbf{y}=\mathbf{H}_{R} \mathbf{x}_{R}+\mathbf{z},
$$

where now $\mathbf{H}_{R}$ is the matrix containing the channel gains from relays to BSs and is defined similarly to $\mathbf{H}$ (see also Fig. 7). We assume per-relay (and thus per-cell) power constraint $\frac{1}{n} \sum_{t=1}^{n}\left|\left[\mathbf{x}_{R}(t)\right]_{m}\right|^{2} \leq Q$, for $m \in[1, M]$.

Consider now the performance of cooperation in cellular networks in the presence of dedicated relay stations, following the uplink model discussed above. Depending on whether one assumes SCP or MCP, the system can be seen as an interference network with relays or as a multiple access channel with multiple relays and a multiple-antenna receiver, respectively. We remark that, in both cases, general conclusive results are unavailable even in the simple two-user cases considered in [39], [64]. Analysis, in terms of achievable per-cell sum-rate and corresponding upper bounds, has been pursued by assuming different transmission strategies and intra-cell TDMA (or equivalently $K=1$ ). Specifically, reference [63] considers half-duplex amplify-and-forward (AF) processing at the relays, [65] studies half-duplex decode-and-forward (DF) relays, [66] full-duplex AF operation, [67] full-duplex DF and [68] full-duplex compress-and-forward, CF. In the following, we briefly review some results for the full duplex case.

In [66], the performance of AF with both SCP and MCP is studied. Relays simply delay the received symbol by at least one time unit, amplify and forward it, sample by sample. Closed-form analytical expressions are obtained for the percell sum-rate based on the observation that the received signal can be seen as the output of a two-dimensional LTI channel via Szego's theorem. The performance of both SCP and MCP is shown to be independent of the time-delay applied by the relays. It is observed that the rates of both schemes are decreasing with the intra-relay interference factor $\mu$. It is also shown that using the full power $Q$ of the relays is unconditionally optimal only for the MCP scheme, while this is not the case with SCP.

In [68], CF relaying with SCP and MCP is studied. Here, the relays operate in blocks, as explained in Sec. III-B, by collecting a number of received samples and compressing the received signal using a vector quantizer. Each BS for SCP, or the CP for MCP, decodes based on the quantized signals received from either the local relay (for SCP) or all the relays (for MCP). For MCP, due to the correlation among the received signals at the relays, distributed compression techniques are applied similarly to [31]. Moreover, the CF scheme with MCP exploits side information available at the $\mathrm{CP}$ regarding the signals transmitted by the relays (which are in fact decoded at the CP). It is proved that the scheme can completely remove the effect of the inter-relay interference. It is noted that, in the nomenclature of the standard IEEE 802.16j [62], both $\mathrm{CF}$ and $\mathrm{AF}$, which are non-regenerative relaying 


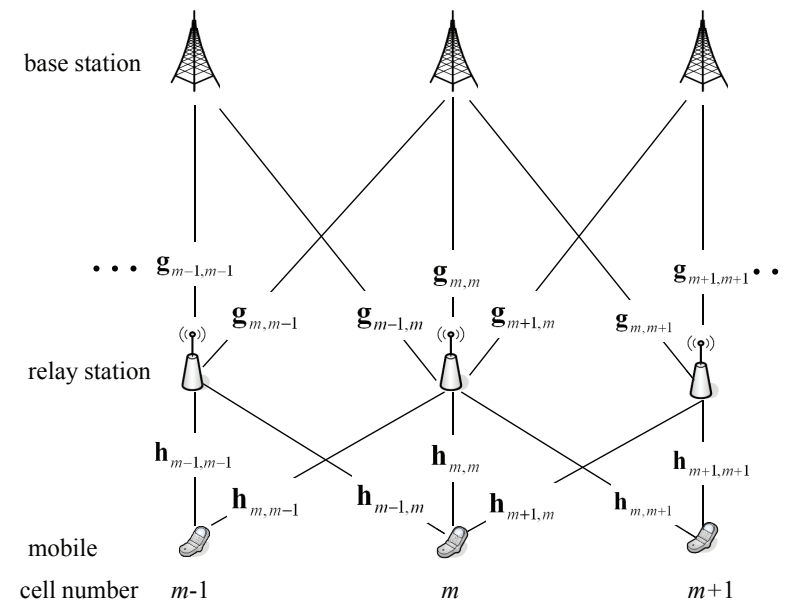

Fig. 7. Relay-aided linear Wyner models with inter-cell interference spans $L_{\ell}=L_{r}=1$ and $K=3$ MSs per cell.

schemes, classify as transparent relaying strategies in that no knowledge of their presence is required at the mobiles. Also, the relays do not require information regarding the codebooks used by the terminals.

Finally, in [67], a regenerative relaying scheme based on DF is considered. Here, codebook information is required at the relays and generally the proposed schemes are nontransparent. The idea is to use rate splitting at the mobile in a similar manner to the standard technique for interference channels [69] so that each relay decodes not only the message of the local mobile (recall that we are assuming intra-cell TDMA), but also part of the message of the adjacent mobiles. This way, the relays can cooperate while transmitting towards the BSs by beamforming the common information.

A comparison among the performance of the schemes described above is shown in Fig. 8 versus the ratio $Q / P$ between the power constraint at the relays $(Q)$ and that at the MSs $(P)$. A first observation is the interplay between SCP or MCP (i.e., cooperation at the BSs) and cooperation via dedicated relays through different strategies. Specifically, it can be seen that if SCP is deployed, DF is advantageous with respect to $\mathrm{CF}$, and also with respect to $\mathrm{AF}$, if the power of the sources is sufficiently larger than that of the relays. It is noted that $\mathrm{CF}$ performs very poorly due to its inability to beamform the users' signals towards the BSs, unlike DF and AF. However, if MCP is in place, the situation is remarkably different in that DF is outperformed by both $\mathrm{CF}$ and $\mathrm{AF}$ unless the sources' power is sufficiently larger than that of the relays. This is because DF is limited by the performance bottleneck due to the need to decode at the relay stations, which prevents the system from benefiting from MCP. Finally, it is seen that the proposed CF scheme performs close to optimal if the relay power is sufficiently large.

We finally recall a different model for cooperation at the mobile level that does not involve dedicated relays but inter-mobile transmission. Namely, [70] models the inter-user

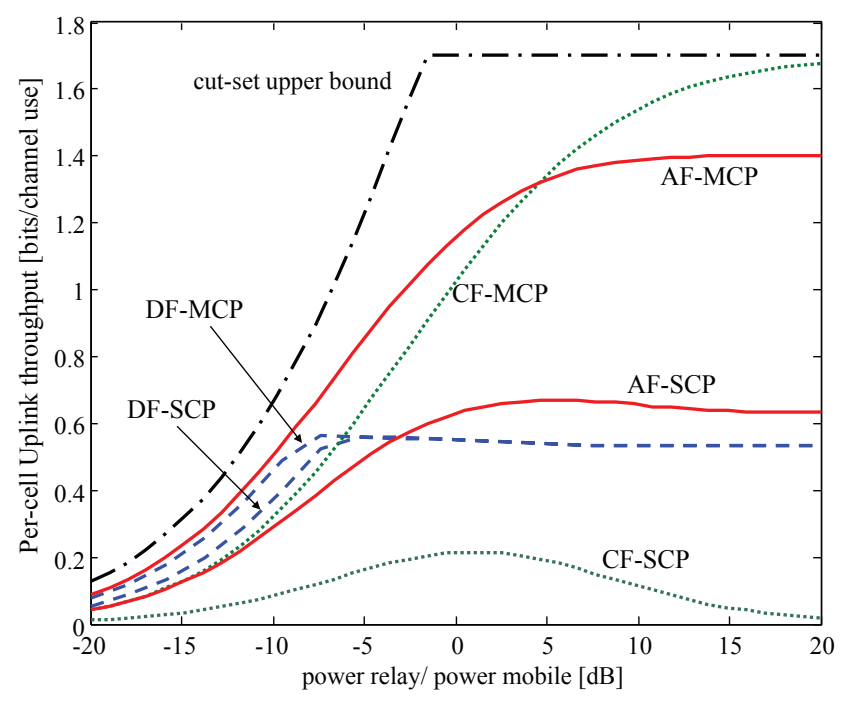

Fig. 8. Per-cell sum-rates achieved by different relaying schemes with SCP or MCP versus the ratio $Q / P$ between the power constraint at the relays $(Q)$ and that at the MSs $(P)$.

links as orthogonal to the main uplink channel, whereas a generalized feedback model (in the sense of [71]) is considered in [67].

\section{F. Conclusions from the Information-Theoretic Models}

This section has illustrated, via information-theoretic arguments, the advantages of cooperation in cellular systems. Cooperation among the $\mathrm{BSs}$ (or $\mathrm{MCP}$ ) has been shown to be able to potentially increase the sum-capacity of the network by an amount proportional to the inter-cell interference span (i.e., number of BSs interfered by a local transmission) with respect to standard single-cell strategies with spatial reuse. While initial work demonstrated such benefits under idealistic conditions, in terms of, e.g., absence of fading and perfect backhaul, more recent research has confirmed the promises of MCP under more practical conditions.

In this section, we have reviewed more recent research that includes practical constraints such as limited backhaul bandwidth, localized base station clustering, and the effect of fading. We conclude that with oblivious BSs there is an $E_{b} / N_{0 \text { min }}$ penalty incurred by limited backhaul, but the capacity is unaffected provided that the backhaul bandwidth scales as $\log$ SNR. Not surprisingly, the impact of BS clustering is not significant provided that the clusters are sufficiently large, see equation (17). On the other hand, the effect of fading is somewhat surprising. As well known for general MIMO links, fading provides the degrees of freedom. But with MCP, it turns out that with a large number of users and BSs, the capacity is increasing in the variance of the fading.

The performance benefits of cooperation at the MS level have been reviewed as well, along with considerations regarding the strong interplay between the design of relaying strategies and MCP techniques. Here, there is much scope for further research, and most of the results reported are of a preliminary nature: Even the simple relay channel is an 
open problem in information theory. But achieveable rates can easily be calculated for particular schemes such as AF, CF, and DF. We conclude that for sufficiently powerful relays, CF is the best technique under MCP. These results show that the MCP model with relays is stimulating new efforts in network information theory.

The presentation has also briefly touched upon the potential gains achievable by exploiting novel transmission strategies such as structured codes. Other advanced techniques, not discussed here, such as interference alignment are also expected to have an important role to play in cooperative cellular systems (see, e.g., [72]). Other related issues of interest are the impact of imperfect channel state information and robust coding strategies [73]. This area remains an active and fertile field for research and is briefly addressed in the next sections.

\section{TRANSMISSION AND CODING TECHNIQUES}

This section provides an overview of transmission and signaling strategies for practical multi-cell MIMO networks, in which the base-stations cooperate. The nature of cooperation (interference coordination or MCP) determines the suitable strategies in various cases. Some of these strategies are straightforward extensions of traditional MIMO signaling techniques, while many others require novel and nontrivial ideas. This section also reviews the possible optimization methods. The optimization space involves scheduling, power allocation, transmit and receive beamforming, as well as choices of transmission strategies. One of the objectives of this section is to highlight the difference between single-cell MIMO techniques and multi-cell techniques. When appropriate, the possible distributed implementation of an algorithm is mentioned, since distributed processing is a primary challenge for the design of multi-cell MIMO networks. However this issue is visited in greater detail in Section V. Below we distinguish between the techniques involving CSI exchange only (interference coordination) and the MCP schemes which require both CSI and user data exchange, and provide an overview of coding, precoding and optimization strategies in each of these cases.

\section{A. Interference coordination strategies}

Consider first a basic level of coordination where only the channel state information of the direct and interfering channels are shared among the BSs, a setup illustrated earlier in Fig. 2. The availability of channel state information allows the transmission strategies across the different cells to adapt to the channel state jointly. Transmission strategies can include scheduling, power control, beamforming, as well as advanced coding methods specifically designed for interference mitigation.

1) Coordinated power control: In an interference-limited cellular network, joint power control and scheduling across the multiple BSs that adapts to the channel condition of the entire network can bring improvement over traditional percell power control. This is especially evident when cellular topology is such that cells significantly overlap.
The resource allocation problem in the multi-cell setting has been studied extensively in the literature [17], [74], [75], [76], [77], [78], [79]. In the following, we consider a simple scenario where both the BS and the remote users are equipped with a single antenna to illustrate the main challenge in multicell power control. In this setting, there is a surprising result for the special case of an arbitrary two-cell set-up where the optimum sum-rate maximizing power allocation policy is in fact binary, i.e. the optimum strategy involves either both cells operating at maximum allowed power or one cell being completely shut down [74]. This result does not extend to more than two cells however.

In a more general setting, consider an orthogonal frequencydivision multiple-access (OFDMA) system in which multiple users within each cell are separated in the frequency domain. Note that the multiple access across the multiple cells is not orthogonal since we allow for full reuse of the frequency tones from one cell to the next. The joint power control and scheduling problem is that of deciding which user should be served and how much power should be used on each frequency tone. Mathematically, in a multi-cell network with $M$ cells, $K$ users per cell, and $N$ OFDM tones, let $h_{l, m, k}^{n}$ denote the channel response between the $l$ th $\mathrm{BS}$ and the $k$ th user in the $m$ th cell in tone $n$. Let $P_{l}^{n}$ denote the power allocation at the $l$ th $\mathrm{BS}$ and $n$th tone. The multi-cell downlink weighted rate maximization problem is

$$
\begin{array}{ll}
\max & \sum_{l=l}^{M} \sum_{k=1}^{K} \alpha_{l k} R_{l k} \\
\text { s.t. } & R_{l k}=\sum_{n \in \mathcal{N}_{l k}} \log \left(1+\frac{P_{l}^{n}\left|h_{l, l, k}^{n}\right|^{2}}{\sum_{j \neq l} P_{j}^{n}\left|h_{j, l, k}^{n}\right|^{2}+1}\right)
\end{array}
$$

where $\mathcal{N}_{l k}$ denotes the set of frequency tones in which the $k$ th user in the $l$ th cell is scheduled. Here, $\alpha_{l k}$ signals the priority of each user, whose value is typically determined by higherlevel protocols, and the background noise variance is assumed to be one without loss of generality. Further, either peak or total power constraints are typically imposed in addition.

Numerically finding the global optimal solution to the above optimization problem is known to be a difficult problem [80]. No convex reformulation of the above problem is known, even in the simpler case of fixed scheduling. In [81], [77], [78], [82], an approach which iterates between scheduling and power allocation has been proposed, but the core difficulty, namely the nonconvexity of the signal-to-interference-andnoise (SINR) expression remains.

One approach for solving the power allocation problem is to let each cell independently optimize its own transmission power in a game theoretical model, where the multiple cells eventually converge to a competitive optimum (e.g., [83], [84], [85]). However, further performance gain can be obtained if cells cooperate.

One idea is to encourage an interfering transmitter to lower its transmit power whenever it causes too much interference to neighboring transmitter-receiver pairs. Toward this end, a promising approach is to devise a mechanism to measure the impact of each transmitter's interference on its neighbors' transmissions, then to coordinate BSs based on the exchange 
of these measures. This idea is called interference pricing, which has been proposed for the power spectrum adaptation problem for the wireless ad-hoc network [86], [87], [88], [89], the digital subscriber line network [90], and is also applicable to wireless multi-cell networks [78], [82]. As shown in these studies, coordinating power control can already yield appreciable improvement in the overall sum rate as compared to a non-coordinated system.

2) Coordinated beamforming: When the BSs are equipped with multiple antennas, the availability of additional spatial dimensions allows the possibility of coordinating beamforming vectors across the BSs, further improving the overall performance. This idea has been explored in [91], [76], [92], [93], [94].

The optimization problem associated with multi-cell joint scheduling, beamforming and power allocation inherits the nonconvex structure of the multi-cell power control problem discussed above. However, there is a particular formulation that enjoys efficient and global optimal solution - this is when the problem is formulated as the minimization of the transmit power across the BSs subject to SINR constraints in a frequency flat channel for the case where the remote users are equipped with a single antenna only. This formulation is most applicable to constant bit-rate applications with fixed quality-of-service constraints.

Let $\mathbf{w}_{l, k}$ be the downlink transmit beamforming vector for the $k$ th user in the $l$ th cell, the downlink SINR for the $k$ th user in the $l$ th cell can be expressed as:

$$
\Gamma_{l, k}=\frac{\left|\mathbf{h}_{l, l, k}^{\dagger} \mathbf{w}_{l, k}\right|^{2}}{\sum_{n \neq k}\left|\mathbf{h}_{l, l, k}^{\dagger} \mathbf{w}_{l, n}\right|^{2}+\sum_{j \neq l, n}\left|\mathbf{h}_{j, l, k}^{\dagger} \mathbf{w}_{j, n}\right|^{2}+1}
$$

where $\mathbf{h}_{j, l, k}$ is now the vector channel from the $j$ th BS to the $k$ th user in the $l$ th cell. Let $\gamma_{l, k}$ be the SINR target for the $k$ th user in the $l$ th cell. We can formulate, for example, a total downlink transmit power minimization problem as follows:

$$
\begin{aligned}
\operatorname{minimize} & \sum_{l=1}^{M} \sum_{k=1}^{K}\left\|\mathbf{w}_{l, k}\right\|^{2} \\
\text { subject to } & \Gamma_{l, k} \geq \gamma_{l, k}, \quad \forall l=1 \cdots M, k=1 \cdots K
\end{aligned}
$$

where the minimization is over the $\mathbf{w}_{l, k}$ 's, which implicitly include both transmit direction and transmit power optimization. For simplicity, we assume that the set of SINR targets are feasible.

Intuitively, coordinating beamforming vectors across the BSs are beneficial when the number of BS antennas exceeds the number of simultaneous users in each cell, in which case the BS has spare spatial dimensions for interference mitigation. In the case where the number of spare dimensions exceeds the number of dominant interferers in every cell, a complete nulling of interference within each cell is possible using a per-cell zero-forcing solution. Insight into the optimal cell loading under coordinated beamforming has been obtained in [95] using large systems analysis.

The key challenge to coordinated beamforming is to coordinate the BSs in such a way as to enable them to find an optimal solution jointly without excessive exchange of channel state information. This turns out to be possible using a tool known as uplink-downlink duality.
The transmit downlink beamforming problem for the multicell system is first considered in the classic work of [96], where an algorithm for iteratively optimizing the beamforming vectors and power allocations is proposed. The key idea is to consider a virtual dual uplink network with transmitters and receivers reversed (so that the uplink channels are the Hermitian transpose of the original downlink channels). The algorithm of [96] proposes to use the optimal uplink receiver beamformers (which are easy to find using the minimum mean-squared error (MMSE) criterion) as the downlink transmit beamformer, then to iterate between the beamformer update step and the power update step to satisfy the target SINRs. The optimality of this algorithm can be established for the single-cell network using several different techniques based on convex optimization methods [97], [98], [99], [100], [101]. In particular, the semidefinite relaxation approach of [98] and the second-order cone programming reformulation of [101] also lead to new and more efficient numerical algorithms for finding the optimal beamformers and powers. Further, it is possible to show that uplink-downlink duality is an example of Lagrangian duality in optimization [59].

The use of convex optimization ideas for establishing duality and for optimal beamforming can be extended to the multicell setting [102], [92]. One consequence of the duality result is that it suggests a way of implementing optimal multi-cell beamforming and power control in a distributed fashion for a time-division duplex (TDD) system, where channel reciprocity guarantees that the actual uplink channels are identical to the virtual dual uplink channels. In this case, the optimal transmit beamformers for the downlink can just simply be set as the MMSE receive beamformers for the uplink. Together with a distributed downlink power control step, this provides a distributed and optimal solution to the problem (25) [92].

The duality result can be further extended to account for the optimization objective of minimizing per-BS or per-antenna powers. The idea is to set up the optimization problem as that of minimizing the weighted sum power, where the weights can be adjusted to tradeoff powers among different BS antennas, and where the weights enter the dual channel as scaling factors for the dual virtual noise variances [59], [92]. In addition, duality also holds for the case where the remote users are equipped with multiple receive antennas as well [103]. However, the iterative updating of transmit beamformer, receive beamformer and the power is no longer guaranteed to converge to the global optimal solution; only a local optimal solution is guaranteed in this case.

Finally, duality holds not only for the power minimization problem formulated in (25), but also for the complementary problem of rate region maximization subject to power constraints (e.g., [104], [105]). This latter problem is of interest in variable rate-adaptive applications. However, as both uplink and downlink networks are MIMO multi-cell interference networks, finding the optimal solution to either problem is a challenging task. In this realm, [106] used an approach based on the first-order condition of the optimization problem, and [107] used a rate profile approach to reach the boundary points of the rate region. In addition, much work has also been done to identify solutions from a competitive (e.g., [108]) or egotistic vs. altruistic points of view [109], [93], [110]. 
Although competitive optimal solutions are not global optimal solutions for the entire network, they nevertheless can offer improvement over existing static networks.

3) Coding for interference mitigation: So far, we have focused on transmission strategies which treat intercell interference as noise. For interference-limited networks, it is possible to further improve these strategies by considering the possibility of detecting then subtracting the interference. In currently deployed cellular networks, interference signals are typically too weak to be detected by out-of-cell users. The key to make interference decoding work is to specifically design transmit signals to facilitate detection at neighboring cells - as suggested by information theoretical results on the interference channel.

The largest known achievable rate region for the two-user interference channel is the celebrated Han-Kobayashi region [69] derived based on the idea of splitting each user's transmit signal into a common message, which is decodable by all receivers, and a private message, which is decodable by the intended receiver only. In other words, by lowering the rate of part of the transmitted message to allow it to be decoded by out-of-cell users, the overall interference level would be reduced, enabling a higher overall rate. The recent work of [111] provides further insights into this scheme by showing that a particular common-private splitting can get within one bit to an outer bound of the two-user interference channel. The key insight is to set the private message power seen at the opposite receiver to be at the background noise level, whereas anything above that should be decoded. Although the outer bound of [111] applies only to the two-user single-antenna case, in a multi-cell MIMO network, adjacent cells can be paired and the optimal beamforming and power splitting problem can be solved together to produce significant performance gain for the overall network [112].

Finally, for an interference channel with more than two transmitters, it is also possible to specifically design transmit signals so that the interferences are always constrained at confined subspaces at each receiver. This allows the receiver to efficiently reject the interference. This idea, known as interference alignment, has been shown to achieve significantly improved multiplexing gain for the MIMO interference network, where both the transmitters and the receivers are equipped with multiple antennas [72]. Practical implementation of these ideas for wireless networks is an active area that is currently attracting much research.

\section{B. Coding strategies for MCP networks}

The coding and optimization strategies considered in the previous sections require the sharing of the channel state information only. Significant further improvement in data rates is possible, if, in addition, the BSs are synchronized and the data streams for all the active users or the received signals at all antennas are shared between the BSs via high-capacity backhaul links [113], [114], [115], [116], [117], [118]. This setup is illustrated in Fig. 3. Many coding strategies have been proposed in the literature for this setting (e.g., [119], [120], [121], [122], [123].) The antennas from all the BSs are in this case effectively pooled together to form a giant antenna array.
The uplink channel can then be modeled as a multiple access channel with multiple transmitters and a single multi-antenna receiver. The downlink channel can be modeled as a broadcast channel with a single multi-antenna transmitter and multiple receivers.

1) Uplink: The capacity region of the uplink multipleaccess channel is achieved with superposition coding and successive decoding [15]. The idea is to decode each user's codeword based on the observation sequence of the entire antenna array (using a linear beamformer across the BSs), then to subtract the decoded codewords in a successive fashion. To achieve this multiple-access channel capacity region, the cooperating BSs theoretically need to share their observation sequence, which requires infinite backhaul capacity.

There is an important special case where the multipleaccess channel capacity can be approximately achieved by just the linear detection of each user's individual message using a receive beamformer across all BSs, without the nonlinear successive decoding step. This happens when the interfering links are much weaker than the direct links (but the interference level is still much stronger than the background noise). Consider the following example where the channel matrix $\mathbf{H}$ between the $K$ single-antenna BSs and $K$ remote users is near diagonal:

$$
\mathbf{y}=\mathbf{H x}+\mathbf{z}
$$

The capacity region of this multiple-access channel is almost a rectangle with each user achieving close to its interferencefree capacity. This is because a joint receiver across the BSs can simply employ a zero-forcing receiver with rows of $\mathbf{H}^{-1}$ as the beamformers. As $\mathbf{H}^{-1}$ is nearly diagonal, it produces minimal noise enhancement. Thus, the single-user interference-free capacity can be nearly achieved for all users with just linear decoding, without the successive decoding step.

Note that for the diagonally dominant network, the above network-wide zero-forcing strategy is superior to an alternative strategy where each BS performs detection based on the received signal at its own antennas only, but BSs share the decoded bits for interference subtraction. In this case, the BSs must follow a particular decoding order with intercell interference subtracted successively. This alternative strategy is clearly suboptimal, because it achieves the single-user interference-free bound only for the last user in any particular decoding order, but not for earlier users. In contrast, the linear strategy mentioned earlier achieves the single-user bound simultaneously for every user in a diagonally dominant interference network.

2) Downlink: The capacity region of the downlink broadcast channel is achieved with a dirty-paper coding strategy at the encoder [14]. The idea is to fix an encoding order, then transmit each user's codeword using a transmit beamformer across all the antennas at all cooperating BSs, and successively encode each user's codeword while treating the messages already encoded as known interference. From an information theoretical point of view, the known interference can be completely pre-subtracted without using extra power at the transmitter. This is called dirty-paper coding [124]. Dirtypaper coding can be approximately implemented in practice 
using Tomlinson-Harashima precoding or lattice precoding strategies (see e.g., [125], [126]).

When the channel matrix associated with the interference network is diagonally dominant, the zero-forcing strategy is again near optimal. Consider again the single-antenna case:

$$
\mathbf{y}=\mathbf{H}^{\dagger} \mathbf{x}+\mathbf{z}
$$

The zero-forcing strategy precodes $\mathbf{x}=\left(\mathbf{H}^{\dagger}\right)^{-1} \mathbf{u}$, where $\mathbf{u}$ is the information symbol. When $\mathbf{H}^{\dagger}$ is near diagonal, it produces minimal power enhancement at the transmitter, resulting in a near rectangular achievable rate region. Note that this network-wide zero-forcing strategy requires joint transmit beamforming across the BSs, but no dirty-paper coding. This is again superior to the alternative strategy of dirty-paper coding without joint beamforming for the diagonally dominant interference network, analogous to the uplink case discussed earlier.

3) Optimization: For a cellular network with an arbitrary topology and a general channel matrix, the optimization of a network-wide beamforming vector together with the successive decoding or dirty-paper precoding orders becomes a relevant question. Consider first the uplink channel:

$$
\mathbf{y}=\sum_{k=1}^{K} \mathbf{H}_{k} \mathbf{x}_{k}+\mathbf{z}
$$

where $\mathbf{y}$ is the network-wide receive signal, and $\mathbf{x}_{k}$ is the transmit signal for user $k$, who may be equipped with multiple antennas as well, and the noise vector $\mathbf{z}$ has a normalized unit variance. Let the optimization problem be formulated as that of maximizing the weighted sum rate $\sum_{k} \alpha_{k} R_{k}$. Because of the polymatroid structure of the multiple-access channel capacity region, the optimal decoding order is completely determined by the relative values of $\alpha_{k}$ [127]. The user with the smallest $\alpha_{k}$ should be decoded first; the user with the largest $\alpha_{k}$ last.

Without loss of generality, let $\alpha_{1} \leq \alpha_{2} \cdots \leq \alpha_{K}$. The resulting weighted sum rate can be expressed as

$$
\sum_{k=1}^{K} \alpha_{k} R_{k}=\sum_{k=1}^{K} \alpha_{k} \log \frac{\operatorname{det}\left(\sum_{j=k}^{K} \mathbf{H}_{j} \mathbf{S}_{j} \mathbf{H}_{j}^{\dagger}+\mathbf{I}\right)}{\operatorname{det}\left(\sum_{j=k+1}^{K} \mathbf{H}_{j} \mathbf{S}_{j} \mathbf{H}_{j}^{\dagger}+\mathbf{I}\right)}
$$

where $\mathbf{S}_{k}$ is the transmit covariance matrix of user $k$. The above rate expression is a convex function of $\mathbf{S}_{k}$. Thus, the weighted sum rate optimization problem for the uplink can be solved efficiently. The eigenvectors of the resulting optimal $\mathbf{S}_{k}$ give the optimal transmit beamformers. The network-wide receive beamformers for user $k$ are the MMSE beamformers with interference from the first $k-1$ users subtracted.

For the downlink channel

$$
\mathbf{y}_{k}=\mathbf{H}_{k}^{\dagger} \mathbf{x}+\mathbf{z}
$$

(where again the noise variance is normalized to one), although a straightforward formulation of the achievable rate region does not result in a convex formulation, a key result known as uplink-downlink duality [128] enables the downlink transmit covariance optimization problem to be translated to the uplink. Uplink-downlink duality guarantees that the capacity region of the downlink channel is identical to the capacity region of the dual uplink, where the transmitters and the receivers are interchanged, and the channel matrices are Hermitian transpose of each other, and where the same sumpower constraint is applied to both. Thus, to find the optimal downlink beamformer, one only needs to solve the optimal uplink problem with a sum power constraint, then use the covariance transformation technique of [128] to translate the optimal uplink solution to the downlink.

The duality result established in [128] solves the optimal downlink beamforming problem with a sum power constraint across all the antennas. In a multi-cell network, the power usages across the BSs cannot easily be traded with each other. In addition, each antenna is typically constrained by the linearity of its power amplifier, and hence is peak power constrained. Thus, a more sensible approach is to apply a perBS or per-antenna power constraint at each cell.

The uplink-downlink duality result can be generalized to accommodate the per-antenna power constraint [59] as mentioned in Section III. The additional ingredient is to recognize that transmit power constraints for the downlink are reflected in the dual uplink as the noise covariances. In particular, for the weighted per-antenna power minimization problem for the downlink, its dual uplink would have its noise variances scaled by the same weights. Further, to enforce per-antenna power constraints, one would need to search over all such weights in the downlink. This amounts to searching over all possible noise variances.

More precisely, for a downlink broadcast channel with perantenna power constraint $P_{i}$ in each of its antennas, the dual uplink is a multiple-access channel with the same sum power constraint $\sum_{i} P_{i}$, but whose noise covariance matrix is a diagonal matrix with $q_{i i}$ on its diagonal and constrained by

$$
\sum_{i} q_{i i} P_{i} \leq \sum_{i} P_{i}
$$

Numerically, the weighted rate sum maximization problem for the downlink becomes a minimax problem in the uplink with maximization over uplink transmit covariances and minimization over uplink receiver noise covariances. This minimax problem is concave in transmit covariance and convex in noise covariance, so it can be solved using convex optimization techniques.

The discussion so far focuses on capacity maximization. When practical coding and modulation schemes are used, an SNR gap needs to be included in the achievable rate computation. Unfortunately, accurate expressions of the SNR gap in the multiuser setting are not easy to obtain. Furthermore, although duality still holds with the inclusion of gap, the dual uplink problem is no longer tractable. The issue is that with an additional gap term, (29) is no longer a concave function of the transmit covariance matrices. Work on finding the approximate optimal ordering and beamformers for the single-receiveantenna case includes [129], but the optimization problem in its full generality remains open.

\section{Coding Strategies with Rate-Limited Cooperation}

In this section, we focus on channel models where the BSs cooperate via rate-limited backhaul links as in Fig. 4-(b), or via independent relay nodes with rate-limited connections to the BSs. These channel models are practically relevant, but the 
information theoretical capacities of these channels are often unknown, except for certain simplified models as mentioned in Section III. This situation is not really surprising considering the fact that the capacity of even the simplest single-transmitter single-receiver and single-relay channel is still open. Thus, instead of capacity analysis, this section focuses on effective interference mitigation techniques in these settings.

1) Receiver Cooperation: In the uplink direction, receiver cooperation can be realized either with a dedicated relay node with fixed-capacity links to the BSs, or with ratelimited conferencing links between BSs which act as relays for each other. In these so-called relay-interference channels, the objective of the relay strategy is typically to mitigate interference, rather than to enhance direct transmission. Wellknown strategies such as decode-and-forward and compressand-forward can both be employed toward this goal.

Consider first a two-user interference channel employing Han-Kobayashi style common-private information splitting. Consider a practical regime of interest where the interfering links are "weak", but where interference is still stronger than background noise. In this case, the rates of the common messages are typically constrained by the interfering links. Thus, when the receivers are equipped with conferencing links, the common message rates can be effectively increased if each receiver decodes the common message from its own transmitter, then forwards a bin index of the common message to the other receiver. Such a decode-and-forward strategy allows each conferencing bit to increase the common information rate (and hence the overall achievable rate) by one bit, up to a limit. This strategy can be shown to be sum capacity achieving in the asymptotic high SNR regime for a simpler Z-interference channel [40]. A more sophisticated coding strategy, which consists of a two-round conferencing with quantization as the first step and binning as the second step, can in fact be proved to be within 2 bits to the capacity region of this channel model for all interference regimes [34].

The decode-and-forward strategy discussed above can be thought of as an interference-forwarding strategy, as the relay decodes and then forwards part of the signal that would have caused interference. The knowledge of the interference can either help the interfered transmit-receive pair subtract the interference, hereby increasing its direct transmission rate, or help the interfering transmitter-receiver pair increase its common message rate. This interference-forwarding strategy has been used in various studies, including interference channel models with a dedicated relay node [130], [131], [132], [133], [134].

In existing multi-cell networks where the Han-Kobayashi style common-private information splitting is not deployed, interference mitigation can be effectively carried out using compress-and-forward or amplify-and-forward strategies. An interesting result in this area is due to the works [135], [136], [137] that show that when a relay observes the precise interference sequence of a transmitter-receiver pair, every relaying bit to the receiver can increase the direct transmission rate by one bit in the noiseless limit. This can be achieved using a compress-and-forward strategy where the relay quantizes its observation of the interference with Wyner-Ziv coding [138], and the receiver first decodes the quantized version of the interference, then subtracts part of the interference before decoding the direct transmission. In fact, the asymptotic optimality of compress-and-forward in the noiseless limit continues to hold when the relay observes a linear combination of the transmitted signal and the interference. This idea can be further extended to show that a single relay can help both transmitter-receiver pairs of an interference channel using a universal strategy called generalized hash-and-forward [139]. Interestingly, although amplify-and-forward is typically not optimal in these settings, the amplify-and-forward strategy can be significantly improved with nonlinear amplification [140].

2) Transmitter Cooperation: In the downlink direction, when the BSs are equipped with rate-limited backhaul links at the transmitter, the BSs can still cooperate using a variety of techniques. One idea is to share part of the common information among the transmitters (assuming a Han-Kobayashi coding strategy is deployed), which allows the transmitters to cooperatively send shared common messages; this idea has been pursued in [141], [142]. Another idea is to share part of the private message, which allows the possibility of partial zero-forcing or dirty-paper coding at the transmitter; these possibilities have been explored in [143], [105], [144], [142]. In certain high SNR and interference-limited asymptotic regimes, it is possible to show that each cooperation bit can improve the direct transmission rate sum by one bit [144]. However, in general, the question of which transmission strategies should be adopted in specific cases remains very much open.

\section{SCALABLE COOPERATIVE SCHEMES}

The potential benefits associated with exploiting or eliminating interference in cellular networks are huge. However, there are several practical hurdles which need to be overcome, over which we now draw the interested reader's attention.

In this section, we address the important issue of scalability. The first models of base station cooperation were centralized in nature, and a natural implementation would consist of a central processing unit, or controller, to which all the base stations are directly connected. The downside to this is that it has a single point of failure and would be an expensive infrastructure to build. Such an architecture would place enormous demands on the back-haul network, as all traffic would have to be routed to and from the central node, causing excessive delays. Besides the problem of user data sharing, there is also the issue of channel state information at the transmitter (CSIT) which also must be shared amongst base stations, and between mobiles and base stations. This is an additional signaling burden associated with MCP. Thus, when it comes to an assessment of the real advantages of MCP in realistic networks, a fundamental question arises: Might it be that the capacity increase due to MCP is outweighed by the signaling overhead it implies?

The information theoretic picture, examined in Section III, reveals that the capacity of the backhaul should grow in proportion to the capacity targeted on the over-the-air section of the network, to avoid being a bottleneck for traffic. Nevertheless, the complete answer to our question seems highly system and scenario dependent and is the focus of ongoing research. A simpler yet related problem would be: how to design practical 
MCP schemes whose overhead scales favorably when the size of the network grows large? This section considers research that has attempted to reduce the overhead required to achieve most of the benefits of cooperation.

One can distinguish two lines of research devoted to overhead reduction. The first deals with deriving efficient representations of the channel state information, which is conveyed to precoding and decoding algorithms. In the second, (perfect or possibly partial) CSI is assumed and attention is focused instead on implementing scalable cooperation schemes via distributed precoding and decoding algorithms. There is not a great deal of difference between trying to obtain efficient channel representations in multi-cell MIMO or in MU-MIMO setups. Since a rich body of literature already exists for this problem, we simply refer the reader to past special journal issues on this topic such as [16], [145]. As a note of caution we point out that existing work on limited CSIT representation for MU-MIMO systems does not take into account the specifics of the multi-cell MIMO channel, such as the different channels from each base station to each user, whose path loss coefficient depends on the user's location in the network. In what follows, we assume that a CSI model already exists at the base stations through feedback channels. We present some concepts related to distributed precoding and decoding and clustering.

\section{A. Impact of channel uncertainty}

1) Network capacity: As discussed in Section III-C5, channel uncertainty affects the scalability of point to point MIMO channels. The number of transmit antennas that can effectively be used is limited by the coherence duration in symbol times. What are the implications for network MIMO? Recently, this issue has been explored in [146], where random matrix theory is exploited to obtain tractable formulas for per-cell rates, involving parameters such as the number of base stations and the coherence duration in symbol times. It is claimed that the per-cell rate can in some cases decrease with the number of base stations, due to the cost of measuring the extra channel parameters. This conclusion may impact the optimal cluster size to use in network MIMO (see Section V-C for a discussion of clustering in the context of MCP). On the other hand, this analysis does not take into account the impact of intercluster interference, leaving open further research on this issue.

2) Downlink: Distributed precoding with partial information sharing: The general problem of distributed multi-cell precoding, whereby the $l$-th base station must design optimally its transmit beamforming vectors on the basis of partially shared CSIT and partially shared user data is largely open. Interestingly, in the case of fully shared user data (MIMO cooperation), this problem can be shown to fall within the framework of team decision theory, which reviews optimization problems in which different agents (here, the base stations) must act cooperatively despite not sharing the same view of the system state [147]. In our context, the problem can be formalized as follows: the users are assumed to feedback their channel state information to all base stations, in a broadcast fashion. As the distance between the user and surrounding bases differ, the quality of feedback for a given channel coefficient is unequal at different base stations.
An optimization problem, by which the beamforming vectors are designed taking into account the locally received CSIT feedback as well as the expected quality level for the feedback received at other bases, is formulated [147]. The obtained beamformers can range smoothly from fully distributed to fully centralized, depending on the feedback model.

Rather than a partial sharing of CSIT along with fully shared user data, another particular framework for distributed precoding assumes a partial sharing of the user data, but under perfect CSIT sharing. A possible practical model for this is as follows: the finite backhaul links are used to convey two types of traffic. The first type is routed in the interferencecoordination mode, i.e. a message to user $k$ in cell $m$ is routed to base station $m$ alone, while the second type of traffic is duplicated to all cooperating bases, in the MIMO fashion. The first and second types are referred to as private and common, respectively. An optimization problem can be formulated by which the total user rate is optimally split across private and common information, as a function of the finite backhaul capacity and of the channel state information [148]. By comparing private and common information rates, one can assess the value of MIMO cooperation depending on the interference strength model.

\section{B. Distributed processing using Turbo Base Stations}

1) Uplink: distributed decoding: We now consider the problem of uplink decoding of multiple base station signals jointly. The fact that the complexity of the general multi-user detection problem grows exponentially with the number of users [149] raises a question of scalability: A priori, it looks as though the multiuser decoding of all users might be intractable as the size of the network grows large. On the other hand, the very localized structure of the interference offers hope of salvation from the apparent intractability, and it motivates the search for decentralized algorithms.

An interesting question to ask is whether the global uplink task of demodulating all the users' data symbols can be distributed across a network of interacting base stations. In this context, each base station is individually performing local computations, and then passing the results to immediate neighbors for further processing. It is very natural to try and apply well known message passing techniques from coding theory, such as the iterative method of Turbo decoding.

A first step in this direction is taken in [150], which considers an uplink multi-user detection (MUD) problem. Each base station first does an independent MUD to try and separate the desired same-cell user from the co-channel interferers in other cells. However, the desired user is also heard at the neighboring base stations, and to gain the benefits of macrodiversity, the base stations share their log-likelihood ratios. The base station controller computes an a posteriori log-likelihood ratio using the log-likelihoods from the local base stations, which is in essence the first step of the Turbodecoder. Later works, such as [151], provided an explicit connection to Turbo-decoding, and propose iterative, message passing algorithms.

A related problem is to find the most likely sequence of bits transmitted in the network. This problem has a simplified 


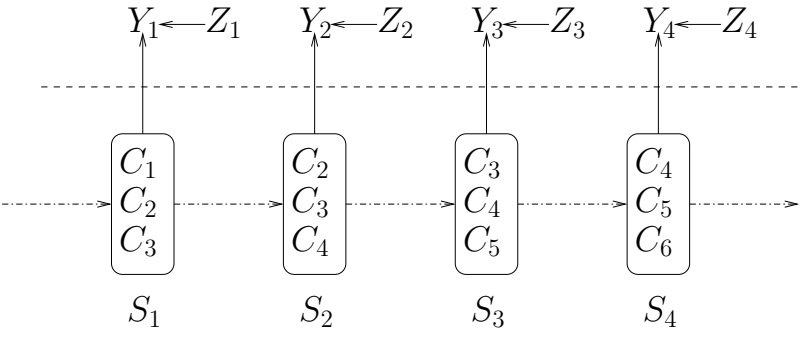

Markov Chain

Fig. 9. Hidden Markov model of linear cellular array

structure due to the local interference coupling, which may make it amenable to a solution via dynamic programming [152].

Turbo decoding is an example of belief propagation on a graph. Communication on the uplink of a one dimensional, linear cellular array model, with one user per cell (as considered in [8], [9]), can be modeled by a Markov chain moving to the right along the linear array, see Figure 9. Each state of the Markov chain corresponds to the choice of code words in three consecutive cells. For example, state $S_{1}$ in Figure 9 consists of the codewords chosen by users 1,2, and 3, respectively. Each base station observes a noisy version of the superposition of the three signals, so it is a hidden Markov model, and a onedimensional probabilistic graph can be associated with this model. The (forward-backward) BCJR algorithm [153] can be applied to compute the MAP estimates of the codewords [154]. This is a one dimensional graphical model, with clustering to provide the Markov structure. Although simple, this model allows an exploration of issues such as distributed computation, parallelism, complexity, and accuracy [154], [155], [156], [157], [158], [30], [159].

Since the complexity of the BCJR grows exponentially with the size of the state space, Gaussian models are considered in [155], [156], where linear estimation techniques are optimal. The analogous problem is Kalman smoothing, and a forwardback Kalman smoother is proposed. Note that the delay and complexity are linear in the network size, but the local nature of the interference can be exploited. In [157] a parallelized version of the forward-backward algorithm is proposed, which allows base stations to make estimates at any time. If the coupling between base stations is weak, or the noise is strong, then accurate estimates are obtained after a small number of message passings. Thus, the complexity and delay, per base station, need not grow with the array size in practice.

More realistic two dimensional cellular array models are more problematic. Forward-backward methods no longer apply, and the associated probabilistic graph models now have loops. The uplink decoding problem is considered in [30], and two graphical models are investigated. The belief propagation algorithm is applied, and it is found that in spite of the loops, error rates near the single user lower bound are obtained, for fading channels. The numerical complexity per base station is a constant, independent of the network size.

Since the complexity of the sum-product algorithm (i.e. belief propagation) grows exponentially with the number of variable nodes connected to a function node, it is of interest to look for suboptimal approaches that reduce the complexity, especially when there are many interfering users per cell. In this case, the computation of the log-likelihood messages sent from a variable node to a function node is in essence an MUD computation. In [160], symbols are grouped, and $a$ posteriori probabilities are computed within a group, treating the interference from the other groups as Gaussian noise, with the mean and variance determined from the a priori probabilities. Thus, a reduced complexity group-wise MUD scheme is proposed. This paper also incorporates an LDPC (Low Density Parity Check) code, so that the graph is in time as well as space.

2) Downlink: Distributed beamforming: The downlink is a broadcast channel in which all base station antennas are pooled. If attention is restricted to linear techniques, then the problem to be solved is that of macroscopic beamforming. As was exposed earlier in Section IV, duality between the uplink and downlink allows some of the above methods to be used on the downlink, also. The downlink beamforming problem can be recast as an equivalent, virtual, uplink estimation problem, in which the downlink data symbols to be transmitted become observables in the uplink problem, and belief propagation on the virtual uplink graph finds the samples to be transmitted by each base station, i.e. the outputs of the global precoder. These samples are obtained by the sum-product message passing algorithm [159].

\section{Limited cooperation via clustering}

Current cellular networks typically connect base transceiver stations (BTS's) to base station controllers (BSC's), and in some implementations the BSC handles the base-band signal processing and encoding/decoding [150]. It is therefore very natural to consider clustered models, in which the processing is done locally at the BSC, which is connected to the adjacent base stations. The collection of base stations served by a BSC forms a cluster, each cluster behaving as a network MIMO system, but now there is interference between adjacent clusters. In this case, there is not a single, centralized node, but many nodes, each independently encoding and decoding signals for the mobiles in the local cluster. The advantages of the clustered model are 1) relevance to currently implemented systems 2) reduced computational complexity 3) reduced demands on the back-haul network since only neighboring base stations (i.e. those which are mutually interfering the most) are engaged in cooperation, and 4) increased robustness to node failures (a base station can be served by more than one system controller). The disadvantages when compared with full network-wide cooperation are 1) increased levels of intercell interference in some areas (since adjacent clusters will interfere), 2) reduced diversity, and 3) lower capacity. Tradeoffs between these factors have been considered in a number of research papers.

It is well known that network MIMO has the capability to eliminate intercell interference. In models in which interference is treated as noise, a notion of effective bandwidths can be developed, which allows a definition of user capacity region [6], [161], [7]. It can be shown that in these models, 


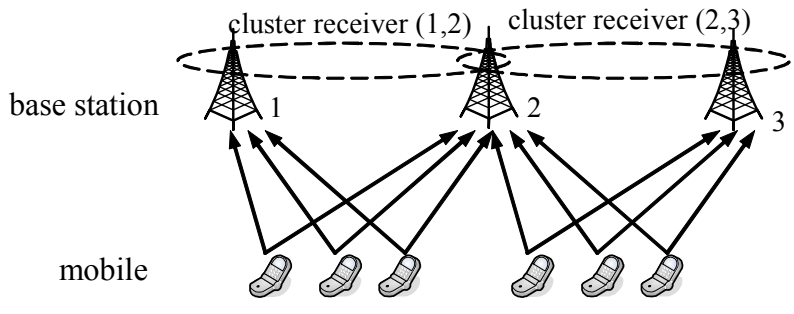

Fig. 10. Cluster decoding using pairs of base stations

base station cooperation and optimal power control effectively eliminate inter-cell interference [162], [5], [6], [7]. In other words, the user capacity region of a network of $K$ cooperating base stations is the same as that of $K$ non-interfering (isolated) cells, as was pointed out in Section III. However, this relies on global cooperation. Consider instead a very simple model consisting of three base stations labelled 1,2 , and 3 , as depicted in Figure 10. Suppose base stations 1 and 2 cooperate to decode the users between them (cluster 1,2), and base stations 2 and 3 cooperate to decode the users between them (cluster 2,3). With full cooperation, there is a capacity limit on the sum of the effective bandwidths in the two cells. With the limited cooperation described here, the user capacity region is reduced, with additional constraints imposed by each cluster. It is shown in Theorem 9.6 in [5] that the user capacity region is the intersection of three regions: one corresponding to each of the two-receiver clusters, and one corresponding to the three-receiver system (the user capacity region under global processing).

Extensions of the above simple three antenna model to more complex networks, including linear and planar models, are considered in [163]. In these models, MCP "receivers" are associated with clusters of antennas, and adjacent receivers share common antennas, as in the simple three antenna model above. In [163] the focus is the information-theoretic capacity, and since the transmitters interfere at nearby receivers, the techniques used come from the theory of interference channels. Upper and lower bounds on achievable rates are derived.

The impact of clustering on the information-theoretic capacity of multi-cell processing (MCP) has been considered more recently in [38], [23], [164], and in other works surveyed in Section III-C4 (i.e. capacity results for the uplink, with local base station backhaul). In [23], the degrees of freedom are shown to be reduced by a factor of $N /(N+1)$ or $(N-1) /(N+1)$, when $N$ is even or odd, respectively, where $N$ is the number of base stations in each cluster. In [38] a similar result is obtained for a limiting regime in which the number of antennas at each base station grows large, in proportion to the number of users in each cell. In this case, the corresponding result is $(N-2) / N$, for $N \geq 3$ : The limiting asymptotics wash out the effect of even or odd parity. Recently, [164] has undertaken a large system analysis of MCP, with clustered decoding, fair user scheduling, and a realistic path loss model.

A practical way to reduce inter-cluster interference is to use frequency planning. For example, one can employ two frequency bands, and by employing appropriate power control in each band, and alternating the roles of each band in adjacent clusters, the impact of inter-cluster interference can be mitigated, whilst maintaining full frequency re-use in each cell [165], [166]. Another approach to clustering is to limit the base station cooperation to the users that really need it i.e. those users near the cell boundaries. The problem then becomes that of grouping users into appropriate clusters for joint MUD. These ideas related to dynamic clustering have started to be investigated in [167], [168] among others.

Clustering reduces the information available to encoders and decoders alike. Information-theoretic approaches in which encoders and decoders are limited to knowledge of the codebooks of users in adjacent cells only are to be found in [154], [41], [37]. Clustering can also create unfairness for mobiles that happen to lie near the boundary of a cluster. A way to treat this problem is to introduce a family of clusters, so that every cell gets a turn at being on a cluster boundary. A round robin across the clusters provides fairness to all the users [38], [165], [166]. Dynamic clustering based on channel strength information also helps to mitigate the unfairness effects in the long run. Clustering has also recently been considered in the context of linear precoding. In [169] mobiles are classified as cluster interior or cluster edge users. Within a cluster, block diagonalization subject to per base station power constraints is performed, but, as in coordinated beamforming, nulls are also steered to neighboring edge users.

\section{REAL-WORLD IMPLEMENTATION AND PERFORMANCE}

The previous sections have addressed the theoretical performance of cooperative networks, including some non-ideal assumptions such as limited backhaul bandwidth and channel uncertainty. In this section, we discuss these and other topics related to the real-world implementation of cooperative techniques in cellular networks. We discuss the practical aspects of system implementation and present system-level simulations and prototypes which hint at the potential and problems of real-world cooperative cellular networks.

\section{A. System implementation}

In the practical implementation of any coherent wireless communication system, issues including synchronization and channel estimation need to be addressed. In addition, downlink MU-MIMO transmission requires channel state information at the base station transmitters, and cooperative networks require an enhanced backhaul network connecting base stations with each other or with a central processor.

1) Synchronization: Downlink MIMO cooperation across multiple base stations requires tight synchronization so that there is ideally no carrier frequency offset (CFO) between the local oscillators at the base stations. Sufficient synchronization could be achieved using commercial GPS (global positioning system) satellite signals for outdoor base stations [170]. For indoor base stations, the timing signal could be sent from an outdoor GPS receiver using a precisely timed network protocol. In the absence of a GPS signal, each base could correct its offset based on CFO estimatation and feedback from the mobiles [171]. On the uplink, CFO results in interference between subcarriers of an OFDM transmission. Techniques for joint detection and CFO compensation in uplink coordinated 
multi-cell MIMO OFDM systems have been proposed in [172].

2) Channel estimation: Coherent combining at the receiver or coherent pre-combining at the transmitter provides SNR gain when the channel state is known. Sufficient resources must be allocated to pilot signals to ensure reliable estimation of the channel state, accounting for the fact that the estimation is performed on each transmit/receive antenna pair with no combining benefit. In the context of network coordination with spatially distributed bases, the extent of the coordination could depend on the range of reliable channel estimation, and there is a tradeoff between increasing the coordination network size at the expense of increased pilot overhead.

For estimation of channels at the transmitter in time-division duplex (TDD) networks, one can rely on the reciprocity of the uplink and downlink channels so that channel estimation on the uplink can be used for downlink transmission. In this sense, channel estimation at the receiver and at the TDD transmitter face similar challenges. However, the TDD system faces additional challenges if the number of users is much larger than the total spatial degrees of freedom, and if the users transmitting uplink data are not the same as those receiving downlink data. Pilot signals and protocols should be designed to address these issues without resulting in excessive training overhead. For example, these issues could be addressed by allowing only high priority users receiving downlink data to transmit uplink pilots, regardless of whether they have uplink data to send.

Estimation of channels at the base station transmitters in frequency-division duplex (FDD) networks face much greater challenges, as mentioned in Section V. In FDD networks, the channel estimates obtained at the mobile receiver must be conveyed to the transmitter, typically over a limited-bandwidth uplink feedback channel. While quantized channel estimates could be fed back, current cellular standards such as LTE [173] implement transmitter "codebooks" consisting of fixed precoding (i.e., beamforming) vectors. Under these standards, the mobile estimates the downlink channel and feeds back an index to its desired precoding vector. In cooperative networks, these codebooks would be designed to contain codeword vectors up to size $M J$, where $M$ is the number of bases and $J$ is the number antennas per base. Because the codebooks for cooperative networks contain more codewords than for conventional networks, additional feedback bits will be required to index the codebooks, most likely leading to an increase in the uplink feedback rate.

Note that the problems for obtaining channel estimates at the transmitter are encountered in single-cell MU-MIMO downlink transmission, but they are more complicated in multi-cell coordinated networks due to the size of the networks and the potential latency introduced in distributing the estimates across the bases.

3) Backhaul issues: Strategies for rate-limited cooperation described in Sections III and V require a high-bandwidth, low-latency backhaul network for connecting the base stations with each other or with a central processor [174][143]. Compared to a conventional network with no coordination, interference coordination techniques shown in Figure 2 require the sharing of channel state information among cooperating bases. MIMO cooperation requires the sharing of both channel state information and user data. As shown in Figure 3, the data symbols of all users must be known at all cooperating bases. With coordination among a cluster of $L$ base stations, the data is sent to these base stations results in a factor of $L$ increase in the backhaul bandwidth. Compared to the exchange of data, the bandwidth required for exchanging channel state information are minimal for the case of moderate mobile speeds [175]. Of course the bandwidth requirements for exchanging channel information increase for higher mobile speeds and more frequency selective channels.

As an alternative to sending the data signals and beamforming weights separately to the bases, one could send a quantized baseband signal. A linearly quantized signal was shown to achieve a significant fraction of the ideal unquantized sumrate performance in an uplink coordinated network [175].

In the context of the 3GPP LTE-Advanced standard [176], network coordination techniques are known as coordinated multi-point (CoMP) transmission or reception. Downlink CoMP transmission requires standardization of signaling and will be addressed as a study item starting in September of 2010 for possible consideration in Release 11 of LTE-Advanced. On the other hand, uplink CoMP reception can be implemented in a proprietary fashion, and could be introduced earlier as a vendor-specific feature.

\section{B. Simulated System Performance}

Network coordination was studied for an indoor network with eight access points arranged in a line and using a TDD framing structure based on WiMAX [177]. Detailed simulations that model the physical layer of the network employed joint zero-forcing precoding and MMSE detection across all access points for the downlink and uplink, respectively. Results confirm that a multiple-fold increase in spectral efficiency is achievable for both the uplink and downlink with conventional channel estimation based on linear interpolation. Interpolation based on minimum mean squared error (MMSE) was also considered but was shown to have nearly identical performance. It is potentially more accurate, but because it requires the estimation of the channel's time-frequency covariance, it is also potentially less robust for higher-speed mobiles.

The downlink cooperative performance of a large multicell FDD network was evaluated in the context of 3GPP LTE parameters [178]. Using pilot signals with powers set according to LTE simulation assumptions, mobiles could not reliably acquire the pilots from multiple base stations, and as a result, cooperation could occur among only a limited set of bases. This was a major limiting factor, reducing the throughput gain by $50 \%$ compared to the ideal theoretical performance. Limited uplink feedback for conveying channel estimates to the base was another important limiting factor, reducing throughput by about $30 \%$. Overall, the performance gains of network coordination in terms of mean throughput were about $20 \%$. These relatively pessimistic results highlight the importance of designing efficient pilot signaling to enable effective channel acquisition and estimation for larger networks and higher mobility users. 


\section{Prototypes and testbeds}

The feasibility of cooperative techniques have been demonstrated in "over-the-air" networks of limited size. A downlink cooperative network with four distributed base antennas serving two users was implemented using zero-forcing precoding [179] as described in Section IV. The proposed system showed significant gains in mean sum-rate capacity (as a function of measured SINR) compared to a conventional time-multiplexed baseline.

Two outdoor testbeds for implementing network coordination have been developed under the EASY-C project (Enablers for Ambient Services and Systems Part C- Cellular networks), a collaboration between academia and industry for the research and development of LTE-Advanced technologies. One testbed in Berlin, Germany, consists of four base station sites (seven sectors) connected through a high-speed optical fiber network [180]. An even larger testbed consists of ten base station sites (28 sectors) distributed in downtown Dresden, Germany [181]. Network coordination has been recently demonstrated over limited portions of each testbed.

Using two distributed base antennas and two users, the Berlin testbed demonstrated downlink network coordination for an FDD LTE trial system [182]. It accounted for many practical implementation aspects including synchronization, CSI uplink feedback, limited modulation and coding schemes, and a finite-bandwidth backhaul connection between the bases. Zero-forcing precoding based on limited CSI feedback was implemented jointly across the two bases. The Dresden testbed demonstrated a similarly detailed field trial for an LTE uplink system, also consisting of two bases and two users [183]. MMSE detection was performed jointly across the bases. In both systems, the users had low mobility (or were stationary), and the systems were isolated so there was no intercell interference. In these relatively benign environments, network coordination was shown to provide significant performance gains over the conventional interference-limited strategy. In particular, it is claimed that MCP can provide median rate gains on the order of at least 50 percent, as well as increased fairness, and improved diversity, taking into account the practical constraints of their system.

Some testbeds are currently testing MCP principles together with the use of relays, in the scenario of so-called mesh networks [184] . Recently a large integrated research project called ARTIST4G funded by the EU and comprising over 20 academic and industrial partners throughout Europe was launched and is fully dedicated to the development of multicell cooperation techniques in future cellular networks. These testbeds and projects allow the exploration of system-level issues discussed in this section as well as broader issues that include hybrid ARQ, resource allocation, and user scheduling.

\section{CONCLUSIONS AND FUTURE DIRECTIONS}

Although the underlying MIMO theoretic concepts are well understood, cooperative systems are still in their infancy and much further research is required in order to fully understand these systems and to practically achieve the full benefits of multi-base cooperation. Unlike standard MIMO systems where the cost of multi-antenna processing lies in the extra hardware and software at individual devices, cooperative MIMO techniques do not necessarily require extra antennas. Rather, the cost lies in the additional exchange of information (user data and channel state) between the devices engaged in the cooperation, or between the devices and the central controller in a centralized architecture. Furthermore, the information exchange is subject to tight delay constraints which are difficult to meet over a large network. MIMO-cooperation offers additional benefits over simpler beamforming coordination schemes, but it requires user data sharing among several BSs and more complex precoding and decoding.

This tutorial began, in Section III, with an extensive review of capacity results for the classical Wyner model and its variants, including limited backhaul bandwidth, localized clustered MCP, and relay assisted MCP. The main conclusions are summarized in Section III-F. Although the Wyner model is mathematically tractable, attention must now steer to more realistic models of cellular communication.

Fading is included in the Wyner model, but the fading parameters are always assumed to be known perfectly at the mobiles and/or base stations. Future work must consider the impact of channel uncertainty, and the cost of measuring the channels in the network. Channel measurement issues may impact the optimal size of clusters in clustered MCP. Bounds on capacity under channel uncertainty are needed, and the coupling of channel uncertainty with limited backhaul bandwidth is an important area yet to be explored. Information-theoretic models provide tractable, elegant capacity formulas that are amenable to optimization, and performance bounds against which practical schemes can be compared. More importantly, however, they provide insights into the key performance bottlenecks, which can then be addressed in more practically oriented research.

Section IV reviewed the transmission and coding techniques required to approach the information-theoretic limits. This included a review of the celebrated uplink-downlink duality theory for the MIMO broadcast channel, which in a rough sense is the model for MCP on the downlink. However, network MIMO has additional constraints, such as limited backhaul bandwidth, the need for decentralized processing, and per base station power constraints. Recent research has included per base station power constraints, and introduced notions such as coordinated beamforming, along with the development of the associated Lagrangian optimization theory. Coordinated beamforming is intermediate between SCP, where only local information is used, and MCP, where global information is available to a central processor. In coordinated beamforming, the BS knows the data and channel state of the users in its own cell, but it also knows the channel state of users in adjacent cells, and this enables a joint optimization problem to be solved.

One challenge for the future is to move these ideas from theory to practice. Joint optimization across many cells may be problematic when channels are changing due to mobility. One approach may be to reformulate the problem in terms of channel statistics, rather than require instantaneous channel knowledge. Another approach may be to look for simplified, suboptimal beamforming structures which nevertheless come close to optimality in practical settings.

Other challenges addressed include coordinated power con- 
trol, and the multi-cell joint problems of scheduling, power control, and rate allocation across the frequency spectrum. Many of these problems are computationally intractable, in general, and the way forward may be to look for structure in real-world networks that allows the problems to be solved in polynomial time. Recent work on fractional frequency reuse in OFDMA systems provides a new set of techniques that could be applied to network MIMO in a joint multicell optimization. Recent work at the cutting edge of network information theory, including interference alignment, network coding, and the recent progress on the interference channel, all provide new ways to approach the fundamental problem: how do we achieve maximum spectral efficiency in a multiple cell network?

Sections V and VI address a few of the fundamental and practical issues, such as scalability, synchronization, and channel estimation. It is highly unlikely that a future network MIMO system will be built according to a centralized architecture. Recent research has considered the problem of distributing the network-wide optimization problems, so that much of the processing can be done locally, with limited communication between nearby nodes. One option is clustered $\mathrm{MCP}$, in which small clusters of BSs collaborate together on uplink decoding and downlink beamforming. Turbo base stations provide another approach, in which soft information is passed between adjacent BSs, allowing iterative, probabilistic graph-based methods to provide decentralized solutions to similar problems. Other interesting approaches lending themselves to distributed implementations are game and team decision theoretic approches.

Behind such problems, a recurrent and quite fundamental issue is associated with the aquisition of channel state information. An important open question is to determine just how much channel state information is needed at each particular node in the network, including information that has been measured at other nodes in the network. This question gives rise to a fundamental feedback resource allocation problem. Cooperation gains go at the expense of feedback resource, hence such a cost is justified when interference is strong enough. More generally, a fundamental trade-off between cooperation and information exchange exists which remains to be explored theoretically.

Another important problem in practice is that of synchronizing the BSs so that there is no carrier frequency offset. GPS offers one approach, but future work may consider methods of distributed clock synchronization. From a practical point of view, distributed precoding and decoding at multiple bases, which are designed to offer cooperation gains while exploiting primarily local channel state and user data information are of high interest and will attract significant research efforts in the years to come.

\section{ACKNOWLEDGEMENTS}

The authors gracefully acknowledge the many constructive comments made by the anonymous reviewers on this paper. David Gesbert acknowledges the partial support of the European Commission seventh framework programme (FP7/20072013) under grant agreements no 247223 (ARTIST4G). Wei
$\mathrm{Yu}$ acknowledges the support of Natural Science and Engineering Research Council (NSERC) of Canada through the Canada Research Chairs Program. Stephen Hanly acknowledges support from a National University of Singapore startup grant.

\section{REFERENCES}

[1] G. Kramer, I. Marić, and R. D. Yates, "Cooperative communications," Found. Trends Netw., vol. 1, no. 3, pp. 271-425, 2006.

[2] D. Gesbert, M. Shafi, P. Smith, D. Shiu, and A. Naguib, "From theory to practice: An overview of MIMO space-time coded wireless systems," IEEE J Sel. Areas Commun., special Issue on MIMO systems, Vol. 21, No. 3, pp. 281-302, April 2003.

[3] A. J. Viterbi, A. M. Viterbi, K. S. Gilhousen, and E. Zehavi, "Soft handoff extends CDMA cell coverage and increases reverse link capacity," IEEE J. Sel. Areas Commun., vol. 12, no. 8, pp. 1281-1288, Oct. 1994.

[4] A. Viterbi, CDMA: Principles of Spread Spectrum Communication. Addison-Wesley, 1995.

[5] S. V. Hanly, "Information capacity of radio networks," Ph.D. dissertation, Cambridge University, Aug. 1993.

[6] - - "Capacity and power control in spread spectrum macrodiversity radio networks," IEEE Trans. Commun., vol. 44, no. 2, pp. 247-256, Feb. 1996

[7] S. V. Hanly and D. N. Tse, "Resource pooling and effective bandwidths in cdma networks with multiuser receivers and spatial diversity," IEEE Trans. Inf. Theory, vol. 47, no. 4, pp. 1328-1351, May 2001.

[8] S. V. Hanly and P. A. Whiting, "Information-theoretic capacity of multi-receiver networks," Telecommunications Systems, vol. 1, no. 1, pp. 1-42, Mar. 1993.

[9] A. D. Wyner, "Shannon-theoretic approach to a Gaussian cellular multiple-access channel," IEEE Trans. Inf. Theory, vol. 40, no. 6, pp. 1713-1727, Nov 1994.

[10] I. E. Telatar, "Capacity of multi-antenna Gaussian channels," AT \& T Bell Laboratories, Tech. Rep. \#BL0112170-950615-07TM, 1995.

[11] G. J. Foschini, "Layered space-time architecture for wireless communication," Bell Labs Technical Journal, vol. 1, no. 2, pp. 41-59, Autumn. 1996.

[12] S. Shamai and B. Zaidel, "Enhancing the cellular downlink capacity via co-processing at the transmitting end," in Vehicular Technology Conference, 2001. VTC 2001 Spring. IEEE VTS 53rd, vol. 3, 2001, pp. 1745-1749 vol.3.

[13] D. Gesbert, M. Kountouris, R. Heath, C.-B. Chae, and T. Salzer, "Shifting the MIMO paradigm," IEEE Signal Processing Mag., Vol. 24, No. 5, pp. 36-46, Sept. 2007.

[14] H. Weingarten, Y. Steinberg, and S. Shamai, "The capacity region of the Gaussian multiple-input multiple-output broadcast channel," IEEE Trans. Inf. Theory, vol. 52, no. 9, pp. 3936-3964, Sept. 2006.

[15] T. M. Cover and J. A. Thomas, Elements of Information Theory, 1 st ed. Wiley, 1991.

[16] D. Love, R. Heath, V. Lau, D. Gesbert, B. Rao, and M. Andrews, "An overview of limited feedback in wireless communication systems," IEEE J. Sel. Areas Commun., Vol. 26, No. 8, pp. 1341-1365, Oct. 2008.

[17] D. Gesbert, S. G. Kiani, A. Gjendemsj, and G. E.Oien, "Adaptation, coordination, and distributed resource allocation in interference-limited wireless networks," Proc. of the IEEE, vol. 95, no. 5, pp. 2393-2409, Dec. 2007.

[18] A. Carleial, "Interference channels," Information Theory, IEEE Transactions on, vol. 24, no. 1, pp. 60-70, Jan 1978.

[19] S. Venkatesan, A. Lozano, and R. Valenzuela, "Network mimo: Overcoming intercell interference in indoor wireless systems," in Signals, Systems and Computers, 2007. ACSSC 2007. Conference Record of the Forty-First Asilomar Conference on, Nov. 2007, pp. 83-87.

[20] G. Caire, S. Ramprashad, H. Papadopoulos, C. Pepin, and C.-E. Sundberg, "Multiuser mimo downlink with limited inter-cell cooperation: Approximate interference alignment in time, frequency and space," in Communication, Control, and Computing, 2008 46th Annual Allerton Conference on, Sept. 2008, pp. 730-737.

[21] O. Somekh, O. Simeone, Y. Bar-Ness, A. M. Haimovich, U. Spagnolini, and S. Shamai, "An information theoretic view of distributed antenna processing in cellular systems," in Distributed Antenna Systems: Open Architecture for Future Wireless Communications. Boston, MA: Auerbach Publications, CRC Press, 2007.

[22] S. Verdu, "The capacity region of the symbol-asynchronous Gaussian multiple-access channel," IEEE Trans. Inf. Theory, vol. 35, no. 4, pp. 733-751, Jul 1989. 
[23] O. Somekh, B. Zaidel, and S. Shamai, "Sum rate characterization of joint multiple cell-site processing," IEEE Trans. Inform. Theory, vol. 53, no. 12, pp. 4473-4497, Dec. 2007.

[24] S. Jing, D. N. C. Tse, J. B. Soriaga, J. Hou, J. E. Smee, and R. Padovani, "Downlink macro-diversity in cellular networks," in Information Theory, 2007. ISIT 2007. IEEE International Symposium on, June 2007, pp. 1-5.

[25] O. Somekh and S. Shamai, "Shannon-theoretic approach to a Gaussian cellular multiple-access channel with fading," Information Theory, IEEE Transactions on, vol. 46, no. 4, pp. 1401-1425, Jul 2000.

[26] N. Levy, O. Zeitouni, and S. Shamai, "On information rates of the fading Wyner cellular model via the thouless formula for the strip," submitted [arXiv:0806.2991v1].

[27] — - "Central limit theorem and large deviations of the fading Wyner cellular model via product of random matrices theory," Problems of Information Transmission, vol. 45, no. 1, pp. 5-22, 2009.

[28] S. Verdu, "Spectral efficiency in the wideband regime," Information Theory, IEEE Transactions on, vol. 48, no. 6, pp. 1319-1343, Jun 2002.

[29] G. Caire, D. Tuninetti, and S. Verdu, "Suboptimality of TDMA in the low-power regime," IEEE Trans. Inf. Theory, vol. 50, no. 4, pp. 608-620, April 2004.

[30] E. Aktas, J. S. Evans, and S. V. Hanly, "Distributed decoding in a cellular multiple access channel," IEEE Transactions on Wireless Communications, vol. 7, no. 1, pp. 241-250, Jan. 2008.

[31] A. Sanderovich, O. Somekh, H. V. Poor, and S. Shamai, "Uplink macro diversity of limited backhaul cellular network," submitted [arXiv:0805.4620].

[32] V. Prabhakaran, D. Tse, and K. Ramachandran, "Rate region of the quadratic Gaussian CEO problem," in Information Theory, 2004. ISIT 2004. Proceedings. International Symposium on, June-2 July 2004, p. 119.

[33] A. E. Gamal, "Achievability for discrete memoryless systems," lecture notes, School of Information Theory, Evanston, IL, Aug. 2009.

[34] I.-H. Wang and D. N. C. Tse, "Interference mitigation through limited receiver cooperation," submitted to IEEE Trans. Inf. Theory, preprint at arXiv:0911.2053, 2009.

[35] O. Simeone, O. Somekh, E. Erkip, S. Shamai, and H. V. Poor, "Multirelay channel with non-ergodic link failures," in Proc. IEEE Workshop on Networking and Information Theory (ITW 2009), June 10-12 2009.

[36] B. Nazer, A. Sanderovich, M. Gastpar, and S. Shamai, "Structured superposition for backhaul constrained cellular uplink," in Proc. IEEE Symposium on Information Theory (ISIT 2009), pp. 1530-1534, June 28 - July 32009.

[37] A. Lapidoth, N. Levy, S. Shamai, and M. A. Wigger, "A cognitive network with clustered decoding," in Proc. IEEE Symposium on Information Theory (ISIT 2009), June 28 - July 3 2009, pp. 596-600.

[38] N. Bacha, J. S. Evans, and S. V. Hanly, "On the capacity of MIMO cellular networks with macrodiversity," in Proc. Australian Communication Theory Workshop, Feb. 2006, pp. 105-109.

[39] O. Sahin, E. Erkip, and O. Simeone, "Interference channel with a relay: Models, relaying strategies, bounds," in Proc. Information Theory and Applications Workshop (ITA 2009), Feb. 8 - 132009.

[40] L. Zhou and W. Yu, "Gaussian Z-interference channel with a relay link: Achievability region and asymptotic sum capacity," submitted to IEEE Trans. Inform. Theory preprint available at arXiv:1006.5087, June 2010.

[41] O. Simeone, O. Somekh, H. V. Poor, and S. Shamai, "Local base station cooperation via finite-capacity links for the uplink of linear cellular networks," IEEE Trans. Inf. Theory, vol. 55, no. 1, pp. 190-204, Jan. 2009.

[42] L. Ozarow, S. Shamai, and A. Wyner, "Information theoretic considerations for cellular mobile radio," IEEE Trans. Veh. Technol., vol. 43, no. 2, pp. 359-378, May 1994.

[43] R. Narasimhan, "Individual outage rate regions for fading multiple access channels," in Proc. IEEE International Symposium on Information Theory. (ISIT 2007). pp. 1571-1575, June 2007.

[44] N. Levy, O. Zeitouni, and S. Shamai, "Information theoretic aspects of users' activity in a Wyner-like cellular model," IEEE Transactions on Information Theory, Vol. 56, No. 5, pp 2241-2248, May 2010.

[45] R. G. Gallager, "An inequality on the capacity region of multiaccess multi-path channels," in Communications and CryptographyTwo Sides of One Tapestry. Norwell, MA: Kluver Academic, 1994, pp. 129-139.

[46] S. Shamai and A. Wyner, "nformation-theoretic considerations for symmetric, cellular, multiple-access fading channels. I," Information
Theory, IEEE Transactions on, vol. 43, no. 6, pp. 1877-1894, Nov 1997.

[47] Y. Liang and A. Goldsmith, "Symmetric rate capacity of cellular systems with cooperative base stations," in Global Telecommunications Conference, 2006. GLOBECOM '06. IEEE, 27 2006-Dec. 1 2006, pp. $1-5$.

[48] O. Somekh, O. Simeone, B. M. Zaidel, H. V. Poor, and S. Shamai, "On the spectrum of large random hermitian finite-band matrices," in Proc. Information Theory and Applications Workshop (ITA 2008), Jan. 27- Feb. 12008.

[49] N. A. Letzepis, "Gaussian cellular multiple access channels," Ph.D. dissertation, Univ. South Australia, 2006.

[50] T. Holliday, A. Goldsmith, and P. Glynn, "Capacity of finite state channels based on Lyapunov exponents of random matrices," IEEE Trans. Inf. Theory, vol. 52, no. 8, pp. 3509-3532, Aug. 2006.

[51] A. M. Tulino and S. Verdú, "Random matrix theory and wireless communications," Now Publishers, 2004.

[52] N. Levy, O. Somekh, S. Shamai, and O. Zeitouni, "On certain large random hermitian jacobi matrices with applications to wireless communications," submitted [arXiv:0806.2674].

[53] A. Lozano, A. Tulino, and S. Verdu, "High-snr power offset in multiantenna communication," IEEE Trans. Inf. Theory, vol. 51, no. 12, pp. 4134-4151, Dec. 2005.

[54] A. Narula, "Information theoretic analysis of multiple-antenna transmission diversity," Ph.D. dissertation, MIT, Cambridge, MA, 1997.

[55] M. Bacha, J. Evans, and S. Hanly, "On the capacity of cellular networks with mimo links," in ICC '06. IEEE International Conference on Communications, vol. 3, June 2006, pp. 1337-1342.

[56] D. Aktas, M. Bacha, J. Evans, and S. Hanly, "Scaling results on the sum capacity of cellular networks with mimo links," IEEE Trans. Inf. Theory, vol. 52, no. 7, pp. 3264-3274, July 2006.

[57] B. Hassibi and B. M. Hochwald, "How much training is needed in multiple-antenna wireless links," IEEE Trans. on Information, vol. 49, no. 4, pp. 951-963, Apr 2003.

[58] G. Caire and S. Shamai, "On the achievable throughput of a multiantenna Gaussian broadcast channel," IEEE Trans. Inf. Theory, vol. 49, no. 7, pp. 1691-1706, July 2003.

[59] W. Yu and T. Lan, "Transmitter optimization for the multi-antenna downlink with per-antenna power constraints," IEEE Trans. Signal Process., vol. 55, no. 6, pp. 2646-2660, June 2007.

[60] O. Simeone, O. Somekh, H. V. Poor, and S. Shamai, "Downlink multicell processing with limited backhaul capacity," EURASIP Journal on Advances in Signal Processing, article ID 840814, Jan. 27- Feb. 1 2009.

[61] O. Somekh, O. Simeone, Y. Bar-Ness, A. Haimovich, and S. Shamai, "Cooperative multicell zero-forcing beamforming in cellular downlink channels," IEEE Trans. Inf. Theory, vol. 55, no. 7, pp. 3206-3219, July 2009.

[62] "http://wirelessman.org/relay/index.html."

[63] O. Simeone, O. Somekh, Y. Bar-Ness, and U. Spagnolini, "Uplink throughput of TDMA cellular systems with multicell processing and amplify-and-forward cooperation between mobiles," IEEE Trans. Wireless Commun., vol. 6, no. 8, pp. 2942-2951, August 2007.

[64] G. Kramer and A. van Wijngaarden, "On the white Gaussian multipleaccess relay channel," in Proc. IEEE International Symposium on Information Theory (ISIT 2000), p. 40, June, 2000.

[65] O. Simeone, O. Somekh, Y. Bar-Ness, and U. Spagnolini, "Throughput of low-power cellular systems with collaborative base stations and relaying," IEEE Trans. Inf. Theory, vol. 54, no. 1, pp. 459-467, Jan. 2008.

[66] O. Somekh, O. Simeone, H. V. Poor, and S. Shamai, "Cellular systems with full-duplex amplify-and-forward relaying and cooperative basestations," in Proc. IEEE International Symposium on Information Theory (ISIT 2007), June 2007, pp. 16-20.

[67] O. Simeone, O. Somekh, G. Kramer, H. V. Poor, and S. Shamai, "Uplink sum-rate analysis of a multi-cell system with feedback," in Proc. Forty-Fifth Annual Allerton Conference on Communication, Control, and Computing, Sept. 23 - 262008.

[68] O. Somekh, O. Simeone, H. Poor, and S. Shamai, "Cellular systems with full-duplex compress-and-forward relaying and cooperative base stations," in Proc. IEEE International Symposium on Information Theory, (ISIT 2008), July 2008, pp. 2086-2090.

[69] T. S. Han and K. Kobayashi, "A new achievable rate region for the interference channel," IEEE Trans. Inf. Theory, vol. 27, no. 1, pp. 4960, Jan. 1981.

[70] O. Simeone, O. Somekh, G. Kramer, H. V. Poor, and S. Shamai, "Throughput of cellular systems with conferencing mobiles and co- 
operative base-stations," Eurasip Journal on Wireless Communications and Networking, article ID 652325, Jan. 2008.

[71] A. Carleial, "Multiple-access channels with different generalized feedback signals," IEEE Trans. Inf. Theory, vol. 28, no. 6, pp. 841-850, Nov 1982.

[72] V. R. Cadambe and S. A. Jafar, "Interference alignment and degrees of freedom of the K-user interference channel," IEEE Trans. Inf. Theory, vol. 54 , no. 8 , pp. $3425-3441$, Aug. 2008

[73] C. W. T. Gou, S. A. Jafar, "On the degrees of freedom of finite state compound wireless networks - settling a conjecture by weingarten et. al," submitted.

[74] A. Gjendemsjoe, D. Gesbert, G. Oien, and S. Kiani, "Binary power control for sum rate maximization over multiple interfering links," IEEE Trans. Wireless Commun., Vol. 7, No. 8, pp. 3164-3173, Aug. 2008.

[75] S. G. Kiani and D. Gesbert, "Optimal and distributed scheduling for multicell capacity maximization," IEEE Trans. Wireless Commun., vol. 7, no. 1, pp. 288-297, Jan. 2008.

[76] M. Vemula, D. Avidor, J. Ling, and C. Papadias, "Inter-cell coordination, opportunistic beamforming and scheduling," in Proc. IEEE Int. Conf. Commun. (ICC), vol. 12, Istanbul, Turkey, June 2006, pp. 5319 5324.

[77] A. L. Stolyar and H. Viswanathan, "Self-organizing dynamic fractional frequency reuse in OFDMA systems," in Proc. IEEE Conf. Comput. Commun. (INFOCOM), Phoenix, AZ, U.S.A., April 2008, pp. 691699.

[78] - - "Self-organizing dynamic fractional frequency reuse for besteffort traffic through distributed inter-cell coordination," in Proc. IEEE Conf. Comput. Commun. (INFOCOM), Rio de Janeiro, Brazil, April 2009, pp. 1287-1295.

[79] V. Tralli, R. Veronesi, and M. Zorzi, "Power-shaped advanced resource assignment (PSARA) for fixed broadband wireless access systems," IEEE Trans. Wireless Commun., vol. 3, no. 6, pp. 2207-2220, Nov. 2004.

[80] Z. Q. Luo and S. Zhang, "Dynamic spectrum management: complexity and duality," IEEE J. Sel. Topics Signal Process., vol. 2, no. 1, pp. 5773, Feb. 2008.

[81] L. Venturino, N. Prasad, and X. Wang, "Coordinated scheduling and power allocation in downlink multicell OFDMA networks," IEEE Trans. Veh. Technol., vol. 58, no. 6, pp. 2835-2848, July 2009.

[82] W. Yu, T. Kwon, and C. Shin, "Joint scheduling and dynamic power spectrum optimization for wireless multicell networks," in Proc. 44th Conf. Info. Science Sys. (CISS), Princeton, NJ, March 2010.

[83] Z. Han, Z. Ji, and K. J. R. Liu, "Non-cooperative resource competition game by virtual referee in multi-cell OFDMA networks," IEEE $J$. Select. Areas Commun., vol. 25, no. 6, pp. 1079-1090, Aug. 2007.

[84] T. Alpcan, T. Basar, and S. Dey, "A power control game based on outage probabilities for multicell wireless data networks," IEEE Trans. Wireless Commun., vol. 5, no. 4, pp. 890-899, April 2006.

[85] A. Leshem and E. Zehavi, "Cooperative game theory and the Gaussian interference channel," IEEE J. Select. Areas Commun., vol. 26, no. 7, pp. 1078-1088, Sept. 2008.

[86] J. Huang, R. A. Berry, and M. L. Honig, "Distributed interference compensation for wireless networks," IEEE J. Select. Areas Commun., vol. 24, no. 5, pp. 1074-1084, May 2006.

[87] J. Yuan and W. Yu, "Distributed cross-layer optimization of wireless sensor networks: A game theoretic approach," in Proc. IEEE Global Telecommun. Conf. (GLOBECOM), San Francisco, U.S.A., 2006.

[88] C. Shi, R. A. Berry, and M. L. Honig, "Distributed interference pricing for OFDM wireless networks with non-separable utilities," in Proc. Conf. Info. Science Sys. (CISS), March 2008, pp. 755-760.

[89] F. Wang, M. Krunz, and S. Cui, "Price-based spectrum management in cognitive radio networks," IEEE J. Sel. Topics Signal Process., vol. 1, no. 2, pp. 74-87, Feb. 2008.

[90] W. Yu, "Multiuser water-filling in the presence of crosstalk," in Information Theory and Applications Workshop, San Diego, U.S.A., Jan. 2007.

[91] R. Veronesi, V. Tralli, J. Zander, and M. Zorzi, "Distributed dynamic resource allocation for multicell SDMA packet access net," IEEE Trans. Wireless Commun., vol. 5, no. 10, pp. 2772-2783, Oct. 2006.

[92] H. Dahrouj and W. Yu, "Coordinated beamforming for the multicell multi-antenna wireless system," IEEE Trans. Wireless Commun., vol. 9, no. 5, pp. 1748-1759, May 2010.

[93] R. Zakhour, Z. K. M. Ho, and D. Gesbert, "Distributed beamforming coordination in multicell MIMO channels," in Proc. IEEE Veh. Tech. Conf. (VTC), Barcelona, Spain, April 2009.
[94] H. Huh, H. C. Papadopoulos, and G. Caire, "Multiuser MIMO transmitter optimization for inter-cell interference mitigation," 2009, preprint: arXiv:0909.1344v1.

[95] R. Zakhour and S. Hanly, "Base station cooperation on the downlink: Large systems analysis," 2010, preprint: arXiv:1006.3360v1.pdf.

[96] F. Rashid-Farrokhi, K. J. R. Liu, and L. Tassiulas, "Transmit beamforming and power control for cellular wireless systems," IEEE $J$. Select. Areas Commun., vol. 16, no. 8, pp. 1437-1450, Oct. 1998.

[97] E. Visotsky and U. Madhow, "Optimal beamforming using transmit antenna arrays," in Proc. IEEE Veh. Technol. Conf. (VTC), vol. 1, July 1999, pp. 851-856.

[98] M. Bengtsson and B. Ottersten, "Optimal and suboptimal transmit beamforming," in Handbook of Antennas in Wireless Communications, L. C. Godara, Ed. CRC Press, 2002.

[99] M. Schubert and H. Boche, "Solution of the multiuser downlink beamforming problem with individual SINR constraints," IEEE Trans. Veh. Technol., vol. 53, pp. 18-28, Jan. 2004.

[100] - - "Iterative multiuser uplink and downlink beamforming under SINR contraints," IEEE Trans. Signal Process., vol. 53, pp. 23242334, July 2005.

[101] A. Wiesel, Y. C. Eldar, and S. Shamai, "Linear precoding via conic optimization for fixed MIMO receivers," IEEE Trans. Signal Process., vol. 54, no. 1, pp. 161-176, Jan. 2006.

[102] R. Stridh, M. Bengtsson, and B. Ottersten, "System evaluation of optimal downlink beamforming with congestion control in wireless communication," IEEE Trans. Wireless Commun., vol. 5, pp. 743-751, April 2006.

[103] B. Song, R. Cruz, and B. Rao, "Network duality for multiuser MIMO beamforming networks and applications," IEEE Trans. Commun., vol. 55, no. 3, pp. 618-630, March 2007.

[104] J. Yang and D. K. Kim, "Multi-cell uplink-downlink beamforming throughput duality based on lagrangian duality with per-base station power constraints," IEEE Commun. Lett., vol. 12, no. 4, pp. 277-279, April 2008.

[105] P. Marsch and G. Fettweis, "On downlink network MIMO under a constrained backhaul and imperfect channel knowledge," in Proc. IEEE Global Telecommun. Conf. (Globecom), Nov. 2009.

[106] L. Venturino, N. Prasad, and X. Wang, "Coordinated linear beamforming in downlink multi-cell wireless networks," IEEE Trans. Wireless Commun., vol. 9, no. 4, pp. 1451-1461, April 2010.

[107] R. Zhang and S. Cui, "Cooperative interference management with MISO beamforming," to appear in IEEE Trans. Signal Processing, 2010, preprint available at arXiv:0910.2771.

[108] G. Scutari, D. Palomar, and S. Barbarossa, "Competitive design of multiuser MIMO systems based on game theory: A unified view," IEEE J. Select. Areas Commun., vol. 26, no. 7, pp. 1089-1103, Sept. 2008.

[109] E. Larsson and E. Jorswieck, "Competition versus cooperation on the MISO interference channel," IEEE J. Select. Areas Commun., vol. 26, no. 9, pp. 1059-1069, Sept. 2008.

[110] K. M. Ho and D. Gesbert, "Balancing egoism and altruism on the interference channel: The mimo case," in Proc. IEEE International Conf. on Communications (ICC), May 2010.

[111] R. H. Etkin, D. N. C. Tse, and H. Wang, "Gaussian interference channel capacity to within one bit," IEEE Trans. Inf. Theory, vol. 54, no. 1, pp. 5534-5562, Dec. 2008.

[112] H. Dahrouj and W. Yu, "Interference mitigation with joint beamforming and common information decoding in multicell systems," in Proc. IEEE Int. Sym. Inf. Theory (ISIT), Austin, TX, June 2010, pp. 2068-2072.

[113] H. Zhang and H. Dai, "Cochannel interference mitigation and cooperative processing in downlink multicell multiuser MIMO networks," EURASIP J. Wireless Commun. Networking, vol. 2004, no. 2, pp. 222 - 235, Dec. 2004, article ID 202654.

[114] H. Zhang, H. Dai, and Q. Zhou, "Base station cooperation for multiuser MIMO: Joint transmission and BS selection," in Proc. Conf. Info. Science Sys. (CISS), Princeton, NJ, March 2004.

[115] S. Jing, D. N. C. Tse, J. B. Soriaga, J. Hou, J. Smee, and R. Padovani, "Multicell downlink capacity with coordinated processing," EURASIP J. Wireless Commun. Networking, vol. 2008, no. 5, Jan. 2008, article ID 586878 .

[116] O. Simeone, O. Somekh, G. Kramer, S. Shamai, and H. V. Poor, "Throughput of cellular systems with conferencing mobiles and cooperative base stations," EURASIP J. Wireless Commun. Networking, 2008, article ID 652325.

[117] M. Karakayali, G. Foschini, and R. Valenzuela, "Network coordination for spectrally efficient communications in cellular systems," IEEE Wireless Commun., vol. 13, no. 4, pp. 56-61, Aug. 2006. 
[118] G. Foschini, M. Karakayali, and R. Valenzuela, "Coordinating multiple antenna cellular networks to achieve enormous spectral efficiency," IEE Proc. Commun., vol. 153, no. 4, pp. 548-555, Aug. 2006.

[119] F. Boccardi and H. Huang, "Limited downlink network coordination in cellular networks," in IEEE Int. Symp. Personal, Indoor and Mobile Radio Commun. (PIMRC), Sept. 2007, pp. 1-5.

[120] S. Venkatesan, "Coordinating base stations for greater uplink spectral efficiency in a cellular network," in IEEE Int. Symp. on Personal, Indoor and Mobile Radio Commun. (PIMRC), Athens, Greece, Sept. 2007, pp. 1-5.

[121] J. Zhang, R. Chen, J. G. Andrews, and R. W. Heath, "Coordinated multi-cell MIMO systems with cellular block diagonalization," in Conf. Record of the Forty-First Asilomar Conf. on Signals, Systems and Computers, Nov. 2007, pp. 1669-1673.

[122] C. Botella, G. Pinero, A. Gonzalez, and M. de Diego, "Coordination in a multi-cell multi-antenna multi-user W-CDMA system: A beamforming approach," IEEE Trans. Wireless Commun., vol. 7, pp. 4479-4485, Nov. 2008.

[123] H. Huang and M. Trivellato, "Performance of multiuser MIMO and network coordination in downlink cellular networks," in Int. Symp. on Modeling and Optimization in Mobile, Ad Hoc, and Wireless Networks (WiOPT), Berlin, April 2008, pp. 85-90.

[124] M. Costa, "Writing on dirty paper," IEEE Trans. Inf. Theory, vol. 29, no. 3, pp. 439-441, May 1983.

[125] U. Erez, S. Shamai, and R. Zamir, "Capacity and lattice strategies for canceling known interference," IEEE Trans. Inf. Theory, vol. 51, no. 1, pp. 3820-3833, Nov. 2005.

[126] W. Yu, D. P. Varodayan, and J. M. Cioffi, "Trellis and convolutional precoding for transmitter-based interference presubtraction," IEEE Trans. Commun., vol. 53, no. 7, pp. 1220-1230, July 2005.

[127] D. N. C. Tse and S. V. Hanly, "Multiaccess fading channels. Part I: Polymatroid structure, optimal resource allocation and throughput capacities," IEEE Trans. Inf. Theory, vol. 44, no. 7, pp. 2796-2815, Nov. 1998.

[128] N. Jindal, S. Vishwanath, and A. Goldsmith, "On the duality of Gaussian multiple-access and broadcast channels," IEEE Trans. Inf. Theory, vol. 50, no. 5, pp. 768-783, May 2004.

[129] C.-H. F. Fung, W. Yu, and T. J. Lim, "Precoding for the multiantenna downlink: Multiuser SNR gap and optimal user ordering," IEEE Trans. Commun., vol. 55, no. 1, pp. 188-197, Jan. 2007.

[130] O. Sahin and E. Erkip, "Achievable rates for the Gaussian interference relay channel," in Proc. Global Telecommun. Conf. (Globecom), Nov. 2007, pp. 1627-1631.

[131] - - "On achievable rates for interference relay channel with interference cancelation," in Conf. Record Forty-First Asilomar Conf. Signals, Systems and Computers, Nov. 2007, pp. 805-809.

[132] O. Sahin, O. Simeone, and E. Erkip, "Interference channel with an outof-band relay," submitted to IEEE Trans. Inf. Theory, 2009, preprint available at arXiv:1007.0267.

[133] I. Marić, R. Dabora, and A. Goldsmith, "On the capacity of the interference channel with a relay," in Proc. IEEE Int. Symp. Inf. Theory (ISIT), pp. 554-558, July 2008

[134] R. Dabora, I. Marić, and A. Goldsmith, "Relay strategies for interference-forwarding," in Proc. IEEE Inf. Theory Workshop (ITW), Porto, Portugal, May 2008, pp. 46-50.

[135] Y.-H. Kim and T. M. Cover, "Capacity of a class of deterministic relay channels," in Proc. IEEE Int. Symp. Inf. Theory (ISIT), June 2007, pp. $591-595$.

[136] Y.-H. Kim, "Capacity of a class of deterministic relay channels," IEEE Trans. Inf. Theory, vol. 53, no. 3, pp. 1328-1329, March 2008.

[137] - -, "Coding techniques for primitive relay channels," in Allerton Conf. Commun. Control and Computing, Sept. 2007.

[138] T. M. Cover and A. El Gamal, "Capacity theorems for the relay channel," IEEE Trans. Inf. Theory, vol. 25, no. 5, pp. 572-584, Sept. 1979.

[139] P. Razaghi and W. Yu, "Universal relaying for the interference channel," in Information Theory and Applications Workshop (ITA), San Diego, U.S.A., Jan. 2010.

[140] M. N. Khormuji, A. Zaidi, and M. Skoglund, "Interference management using nonlinear relays," IEEE Trans. Commun., vol. 58, no. 7, pp. 1924-1930, July 2010.

[141] I. Marić, R. D. Yates, and G. Kramer, "Capacity of interference channels with partial transmitter cooperation," IEEE Trans. Inf. Theory, vol. 53, no. 1, pp. 3536-3548, Oct. 2007.

[142] H. Bagheri, A. S. Motahari, and A. K. Khandani, "Zero-forcing for the symmetric interference channel with conferencing encoders," in Proc. IEEE Int. Symp. Inf. Theory (ISIT), Austin, TX, June 2010, pp. 370-374.
[143] P. Marsch and G. Fettweis, "On base station cooperation schemes for downlink network MIMO under a constrained backhaul," Proc. IEEE Global Telecommunications Conference (Globecom'08), Dec. 2008.

[144] S. Hari and W. Yu, "Partial zero-forcing precoding for the interference channel with partially cooperating transmitters," in Proc. IEEE Int. Symp. Inf. Theory (ISIT), Austin, TX, June 2010, pp. 2283-2287.

[145] R. Heath, D. Love, V. Lau, D. Gesbert, B. Rao, and M. Andrews (Editors), "Exploiting limited feedback in tomorrow's communication networks," Special Issue of IEEE J. Sel. Areas Commun., Vol. 26, No. 8, pp. 1337-1340, Oct. 2008.

[146] J. Hoydis, M. Kobayashi, and M. Debbah, "On the optimal number of cooperative base stations in network MIMO systems," 2010, preprint: arXiv: $1003.0332 \mathrm{v} 1$.

[147] R. Zakhour and D. Gesbert, "Team decision for the cooperative MIMO channel with imperfect CSIT sharing," in The Information Theory and Applications (ITA) Workshop, San Diego CA., Feburary 2010.

[148] - - "On the value of data sharing in constrained-backhaul network MIMO," in Proc. International Zurich Seminar on Communications, March 2010.

[149] S. Verdú, Multiuser Detection. Norwell, MA: Cambridge University Press, 1998.

[150] M. C. Valenti and B. D. Woerner, "Iterative multiuser detection, macrodiversity combining, and decoding for the TDMA cellular uplink," IEEE J. Sel. Areas Commun., vol. 19, pp. 1570-1583, Aug. 2001.

[151] T. Mayer, H. Jenkac, and J. Hagenauer, "Turbo base-station cooperation for intercell interference cancellation," in Proc. IEEE International Conference on Communications, vol. 11, June 2006, pp. 4977 - 4982.

[152] L. R. Welburn, J. K. Cavers, and K. W. Sowerby, "A computational paradigm for space-time multiuser detection," IEEE Trans. Commun., vol. 52, no. 9, pp. 1595-1604, Sep. 2004.

[153] L. Bahl, J. Cocke, F. Jelinek, and J. Raviv, "Optimal decoding of linear codes for minimizing symbol error rate," IEEE Trans. Inf. Theory, vol. 20, no. 2, pp. 284-287, Mar. 1974.

[154] A. J. Grant, S. V. Hanly, J. S. Evans, and R. Müller, "Distributed decoding for Wyner cellular systems," in Proc. 5th Australian Communications Theory Workshop, Newcastle, Australia, Feb. 2004, pp. 77-81.

[155] B. L. Ng, J. S. Evans, S. V. Hanly, and A. J. Grant, "Information capacity of Wyner's cellular network with LMMSE receivers," in Proc. IEEE International Conference on Communications, Paris, France, vol. 1, June 2004, pp. 583 - 587.

[156] - -, "Distributed linear multiuser detection in cellular networks," in Proc. 5th Australian Communications Theory Workshop, Newcastle, Australia, Feb. 2004, pp. 127-132.

[157] B. L. Ng, J. S. Evans, and S. V. Hanly, "Distributed linear multiuser detection in cellular networks based on Kalman smoothing," in Proc. IEEE Global Telecommunications Conference, Dallas, USA, vol. 1, Dec. 2004, pp. $134-138$.

[158] - - , "Distributed downlink beamforming in cellular networks," in Proc. IEEE International Symposium on Information Theory, Jun. 2007, pp. 6-10.

[159] B. L. Ng, J. S. Evans, S. V. Hanly, and D. Aktas, "Distributed downlink beamforming with cooperative base stations," IEEE Trans. Inf. Theory, vol. 54, no. 12, pp. 5491-5499, Dec. 2008.

[160] S. Bavarian and J. K. Cavers, "Reduced-complexity belief propagation for system-wide mud in the uplink of cellular networks," IEEE J. Sel. Areas Commun., vol. 26, no. 3, pp. 541-549, Apr. 2008.

[161] D. N. Tse and S. V. Hanly, "Linear multi-user receivers: Effective interference, effective bandwidth, and user capacity," IEEE Trans. Inf. Theory, vol. 45, no. 2, pp. 641-657, Mar. 1999.

[162] S. V. Hanly, "Macro-diversity for spread spectrum mobile radio: Capacity and power control," in Proc. IEEE Second International Symposium on Spread Spectrum Techniques and Applications, Nov. 1992, pp. 349-352.

[163] N. Levy and S. S. (Shitz), "Clustered Local Decoding for Wyner-Type Cellular Models," IEEE Trans. Inf. Theory, vol. 55, no. 11, pp. 49674985, November 2009.

[164] H. Huh, S. H. Moon, Y. T. Kim, I. Lee, and G. Caire, "Multicell MIMO downlink with cell cooperation and fair scheduling: A large system analysis," 2010, preprint: arXiv:1006.2162v1.pdf.

[165] G. Caire, S. Ramprashad, H. Papadopoulos, C. Pepin, and C.-E. W. Sundberg, "Multiuser MIMO Downlink with Limited Inter-cell Cooperation: Approximate Interference Alignmnent in Time, Frequency and Space," in Proc. 46th Annual Allerton Conference on Communication, Control, and Computing, September 2008, pp. 730-737.

[166] E. Katranaras, M. Imran, and R. Hoshyar, "Sum-rate of linear cellular systems with clustered joint processing," Vehicular Technology Conference-VTC 2009, Spring, 2009, vol. 8, no. 4, pp. 1910-1921. 
[167] P. Marsch and G. Fettweis, "A framework for optimizing the uplink performance of distributed antenna systems under a constrained backhaul," in Proc. IEEE International Conference on Communications, June 2007, pp. 975-979.

[168] A. Papadogiannis, D. Gesbert, and E. Hardouin, "A dynamic clustering approach in wireless networks with multi-cell cooperative processing," in Proc. IEEE Intern. Conf. on Comm. (ICC), pp. 4033-4037, May 2008.

[169] J. Zhang, R. Chen, J. G. Andrews, A. Ghosh, and R. W. Heath, "Networked mimo with clustered linear precoding," IEEE Trans. Wireless Commun., Vol. 8, No. 4, pp. 1910-1921, April 2009.

[170] V. Jungnickel, T. Wirth, M. Schellmann, T. Haustein, and W. Zirwas, "Sychronization of cooperative base stations," Proc. International Symposium on Wireless Communication Systems (ISWCS'08), October 2008.

[171] B. Zarikoff and J. Cavers, "Multiple frequency offset estimation for the downlink of coordinated MIMO systems," IEEE J. Sel. Areas Commun., vol. 26, pp. 901-912, 2008.

[172] V. Kotzsch, J. Holfeld, and G. Fettweis, "Joint detection and CFO compensation in asynchronous multi-user MIMO OFDM systems," Proc. 69th IEEE Vehicular Technology Conference (VTC'09), vol. 2009, April 2009.

[173] S. Sesia, I. Toufik, and M. Baker, Eds., LTE: The UMTS Long Term Evolution. John Wiley and Sons, 2009.

[174] P. Marsch and G. Fettweis, "On uplink network MIMO under a constrained backhaul and imperfect channel knowledge," Proc. IEEE International Conference on Communications (ICC'09), 2009.

[175] D. Samardzija and H. Huang, "Determining backhaul bandwidth requirements for network MIMO," Proc. 17th European Signal Processing Conference (EUSIPCO'09), 2009.

[176] B. Clerckx, A. Lozano, S. Sesia, C. van Rensburg, and C. B. Papadias, "3GPP LTE and LTE Advanced," EURASIP Journal on Wireless Communications and Networking, 2009.

[177] S. Venkatesan, H. Huang, A. Lozano, and R. Valenzuela, "A WiMAXbased implementation of network mimo for indoor wireless systems," Eurasip Journal on Advances in Signal Processing, special issue on Multiuser MIMO transmission with limited feedback, cooperation and coordination, no. Article ID 963547, 2009.

[178] S. Annapureddy, A. Barbieri, S. Geirhofer, S. Mallik, and A. Gorokhov, "Coordinated joint transmission in WWAN," IEEE Communication Theory Workshop, 2010.

[179] D. Samardzija, H. Huang, T. Sizer, and R. Valenzuela, "Experimental downlink multiuser MIMO system with distributed and coherentlycoordinated transmit antennas," Proc. International Conference on Communications (ICC'09), June 2007.

[180] V. Jungnickel, L. Thiele, M. Schellmann, T. Wirth, T. Haustein, O. Koch, W. Zirwas, and E. Schulz, "Interference aware scheduling in the multiuser MIMO-OFDM downlink," IEEE Commun. Mag., vol. 47, no. 6 , pp. $56-66$, June 2009.

[181] R. Irmer, H.-P. Mayer, A. Weber, V. Braun, M. Schmidt, M. Ohm, N. Ahr, A. Zoch, C. Jandura, P. Marsch, and G. Fettweis, "Multisite field trial for LTE and advanced concepts," IEEE Commun. Mag., vol. 47, no. 2, pp. 92 - 98, February 2009.

[182] V. Jungnickel, A. Forck, S. Jaeckel, F. Bauermeister, S. Schiffermueller, S. Schubert, S. Wahls, L. Thiele, T. Haustein, W. Kreher, J. Mueller, H. Droste, and G. Kadel, "Field trials using coordinated multi-point transmission in the downlink," Proc. 3rd Int. Workshop on Wireless Distributed Networks (WDN), held in conjunction with IEEE PIMRC, 2010.

[183] M. Grieger, P. Marsch, Z. Rong, and G. Fettweis, "Field trial results for a coordinated mutli-point (CoMP) uplink in cellular systems," Proc. ITG/IEEE Workshop on Smart Antennas (WSA'10), 2010.

[184] "The openair wireless experimentation platform," in Available under http://www.openairinterface.org/.

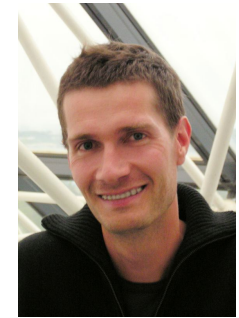

David Gesbert (IEEE SM) is Professor in the Mobile Communications Dept., EURECOM, France. $\mathrm{He}$ obtained the Ph.D degree from Ecole Nationale Superieure des Telecommunications, France, in 1997. From 1997 to 1999 he has been with the Information Systems Laboratory, Stanford University. In 1999, he was a founding engineer of Iospan Wireless Inc, San Jose, Ca.,a startup company pioneering MIMO-OFDM (now Intel). Between 2001 and 2003 he has been with the Department of Informatics, University of Oslo as an adjunct professor. D. Gesbert has published about 170 papers and several patents all in the area of signal processing, communications, and wireless networks.

D. Gesbert was a co-editor of several special issues on wireless networks and communications theory, for JSAC (2003, 2007, 2010), EURASIP Journal on Applied Signal Processing (2004, 2007), Wireless Communications Magazine (2006). He served on the IEEE Signal Processing for Communications Technical Committee, 2003-2008. He's an associate editor for IEEE Transactions on Wireless Communications and the EURASIP Journal on Wireless Communications and Networking. He authored or co-authored papers winning the 2004 IEEE Best Tutorial Paper Award (Communications Society) for a 2003 JSAC paper on MIMO systems, 2005 Best Paper (Young Author) Award for Signal Proc. Society journals, and the Best Paper Award for the 2004 ACM MSWiM workshop. He co-authored the book "Space time wireless communications: From parameter estimation to MIMO systems", Cambridge Press, 2006

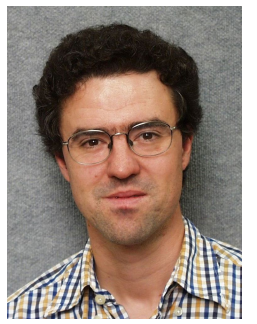

Stephen Hanly (M'98) received a B.Sc. (Hons) and M. Sc. from the University of Western Australia, and the Ph.D. degree in mathematics in 1994 from Cambridge University, UK. From 1993 to 1995, he was a Post-doctoral member of technical staff at AT\&T Bell Laboratories. From 1996 to 2009 he was on the research and teaching staff at the University of Melbourne. He is presently an Associate Professor in the Department of Electrical and Computer Engineering at the National University of Singapore. He was an Associate Editor for IEEE Transactions on Wireless Communications from 2005-2009, and is guest editor for the IEEE Journal on Selected Areas special issue on "Cooperative Communications in MIMO Cellular Networks". In 2005, he was the technical co-chair for the IEEE International Symposium on Information Theory held in Adelaide, Australia. He was a co-recipient of the best paper award at the Infocom 1998 conference, and the 2001 Joint IEEE Communications Society and Information Theory Society best paper award, both for his work with David Tse (Berkeley). His research interests are in the areas of information theory, signal processing, and wireless networking.

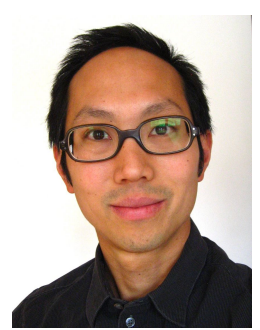

Howard Huang received a BSEE degree from Rice University in 1991 and a Ph.D. in electrical engineering from Princeton University in 1995. Since then, he has been a researcher at Bell Labs, in Holmdel, New Jersey, currently as a Distinguished Member of Technical Staff in the wireless access domain of Alcatel-Lucent. His research interests include wireless communication theory and cellular system design. He has taught as an adjunct professor at Columbia University and is a Senior Member of the IEEE. 


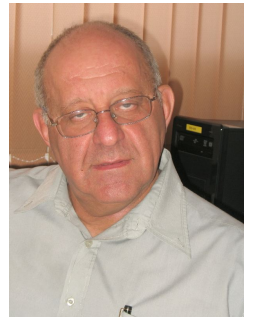

Shlomo Shamai Shitz received the B.Sc., M.Sc., and $\mathrm{Ph} . \mathrm{D}$. degrees in electrical engineering from the Technion-Israel Institute of Technology, in 1975, 1981 and 1986 respectively.

During 1975-1985 he was with the Communications Research Labs in the capacity of a Senior Research Engineer. Since 1986 he is with the Department of Electrical Engineering, TechnionIsrael Institute of Technology, where he is now the William Fondiller Professor of Telecommunications. His research interests encompasses a wide spectrum of topics in information theory and statistical communications.

Dr. Shamai Shitz is an IEEE Fellow, and the recipient of the 2011 Claude E. Shannon Award. He is the recipient of the 1999 van der Pol Gold Medal of the Union Radio Scientifique Internationale (URSI), and a co-recipient of the 2000 IEEE Donald G. Fink Prize Paper Award, the 2003, and the 2004 joint IT/COM societies paper award, the 2007 IEEE Information Theory Society Paper Award, the 2009 European Commission FP7, Network of Excellence in Wireless COMmunications (NEWCOM++) Best Paper Award, and the 2010 Thomson Reuters Award for International Excellence in Scientific Research. $\mathrm{He}$ is also the recipient of 1985 Alon Grant for distinguished young scientists and the 2000 Technion Henry Taub Prize for Excellence in Research. He has served as Associate Editor for the SHANNON THEORY OF THE IEEE TRANSACTIONS ON INFORMATION THEORY, and has also served on the Board of Governors of the Information Theory Society.

Osvaldo Simeone received the M.Sc. degree (with honors) and the Ph.D. degree in Information Engineering from Politecnico di Milano, Milan, Italy, in 2001 and 2005 respectively. He is currently with the Center for Wireless Communications and Signal Processing Research (CWCSPR), at the New Jersey Institute of Technology (NJIT), Newark, New Jersey, where he is an Assistant Professor. His current research interests concern the cross-layer analysis and design of wireless networks with emphasis on information-theoretic, signal processing and queuing aspects. Specific topics of interest are: cognitive radio, cooperative communications, ad hoc, sensor, mesh and hybrid networks, distributed estimation and synchronization. Dr. Simeone is the co-recipient of the best paper awards of IEEE SPAWC 2007 and IEEE WRECOM 2007. He currently serves as an Editor for IEEE Trans. Commun.

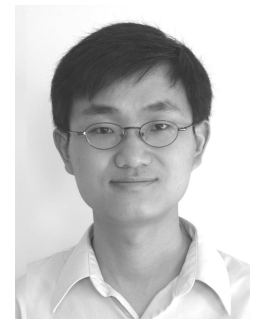

Wei Yu (S'97-M'02-SM'08) received the B.A.Sc. degree in Computer Engineering and Mathematics from the University of Waterloo, Waterloo, Ontario, Canada in 1997 and M.S. and Ph.D. degrees in Electrical Engineering from Stanford University, Stanford, CA, in 1998 and 2002, respectively. Since 2002, he has been with the Electrical and Computer Engineering Department at the University of Toronto, Toronto, Ontario, Canada, where he is now an Associate Professor and holds a Canada Research Chair in Information Theory and Digital Communications. His main research interests include multiuser information theory, optimization, wireless communications and broadband access networks.

Prof. Wei $\mathrm{Yu}$ is currently an Editor for IEEE TRANSACTIONS ON COMMUNICATIONS. He was an Editor for IEEE TRANSACTIONS ON WIRELESS COMMUNICATIONS from 2004 to 2007, and a Guest Editor for a number of special issues for the IEEE Journal on SELECTEd AREAS IN COMMUNICATIONS and the EURASIP JOURNAL ON APPLIED SignAl PROCESSING. $\mathrm{He}$ is member of the Signal Processing for Communications and Networking Technical Committee of the IEEE Signal Processing Society. He received the IEEE Signal Processing Society Best Paper Award in 2008, the McCharles Prize for Early Career Research Distinction in 2008, the Early Career Teaching Award from the Faculty of Applied Science and Engineering, University of Toronto in 2007, and the Early Researcher Award from Ontario in 2006. 